\title{
ER-resident sensor PERK is essential for mitochondrial thermogenesis in brown adipose tissue
}

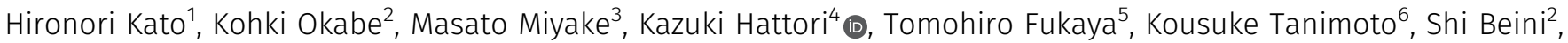 \\ Mariko Mizuguchi ${ }^{7}$, Satoru Torii ${ }^{8}$, Satoko Arakawa ${ }^{8}$, Masaya Ono ${ }^{9}$, Yusuke Saito ${ }^{10}$, Takashi Sugiyama ${ }^{1}$, Takashi Funatsu ${ }^{2}$ (D, \\ Katsuaki Sato ${ }^{5}$, Shigeomi Shimizu ${ }^{8}$, Seiichi Oyadomari ${ }^{3}$, Hidenori Ichijo ${ }^{4}$, Hisae Kadowaki ${ }^{1}$, Hideki Nishitoh ${ }^{1}$ (®
}

\begin{abstract}
Mitochondria play a central role in the function of brown adipocytes (BAs). Although mitochondrial biogenesis, which is indispensable for thermogenesis, is regulated by coordination between nuclear DNA transcription and mitochondrial DNA transcription, the molecular mechanisms of mitochondrial development during BA differentiation are largely unknown. Here, we show the importance of the ERresident sensor PKR-like ER kinase (PERK) in the mitochondrial thermogenesis of brown adipose tissue. During BA differentiation, PERK is physiologically phosphorylated independently of the ER stress. This PERK phosphorylation induces transcriptional activation by GA-binding protein transcription factor $\alpha$ subunit (GABP $\alpha$ ), which is required for mitochondrial inner membrane protein biogenesis, and this novel role of PERK is involved in maintaining the body temperatures of mice during cold exposure. Our findings demonstrate that mitochondrial development regulated by the PERKGABP $\alpha$ axis is indispensable for thermogenesis in brown adipose tissue.
\end{abstract}

DOI 10.26508/lsa.201900576 | Received 9 October 2019 | Revised 22 January 2020 | Accepted 23 January 2020 | Published online 6 February 2020

\section{Introduction}

Brown adipose tissue (BAT) is one of the major tissues causing nonshivering thermogenesis in homeothermic animals exposed to cold stress and plays an important role in metabolic function that contributes to energy consumption (Cannon \& Nedergaard, 2004). Thermogenesis in brown adipocytes (BAs) is mediated by the function of uncoupling protein 1 (UCP1), which localizes to the mitochondrial inner membrane and dissipates the mitochondrial proton electrochemical gradient (Susulic et al, 1995; Matthias et al, 2000; Feldmann et al, 2009). The development of BAs consists of two steps: lineage commitment from precursor cells to brown preadipocytes and differentiation from brown preadipocytes into mature BAs (Harms \& Seale, 2013; Kajimura \& Saito, 2014). Differentiated BAs have unique morphological characteristics; these cells possess multiple lipid droplets (LDs) and a number of expanded mitochondria that contain dense parallel cristae (Napolitano \& Fawcett, 1958). The highly developed cristae are effective in maintaining the mitochondrial membrane potential $(\Delta \psi \mathrm{m})$, which is essential for two main functions: oxidative phosphorylation (OXPHOS)-dependent ATP production, which mainly occurs in LD-associated mitochondria, and thermogenesis mediated by cytoplasmic-free mitochondria (Benador et al, 2018). However, the mechanism by which BAs acquire these developed mitochondria remains unknown.

Some areas on the mitochondrial surface make close contact with the ER membrane in various types of cells (Kato \& Nishitoh, 2015). ER-mitochondria contact dynamically fluctuates in response to various types of stimuli and regulates a number of cellular functions, such as calcium homeostasis (Rizzuto et al, 1998; Hirabayashi et al, 2017), lipid biosynthesis (Kornmann et al, 2009), mitochondrial dynamics regulated by fusion and fission (Friedman et al, 2011), and autophagy (Hamasaki et al, 2013). Although the ER in differentiated BAs is not as developed as it is in other secretory cells, a large area of the ER membrane in BAs attaches to the mitochondrial outer membrane (de Meis et al, 2010; Golic et al, 2014), and ER-resident molecules contribute to mitochondrial biogenesis (Bartelt et al, 2018; Zeng et al, 2019). However, the molecular mechanism by which ER-mitochondria crosstalk regulates the functions of BAs remains unclear.

In mammalian cells, three types of ER-resident stress sensors, PKR-like ER kinase (PERK), inositol-requiring enzyme $1 \alpha$ (IRE1 $\alpha$ ), and activating transcription factor (ATF) 6, are activated by ER stress, resulting in activation of the unfolded protein response (UPR). Under ER stress conditions, activation of PERK is triggered by the dissociation of glucose-regulated protein (GRP) 78 (also known as

\footnotetext{
${ }^{1}$ Laboratory of Biochemistry and Molecular Biology, Department of Medical Sciences, University of Miyazaki, Miyazaki, Japan ${ }^{2}$ Laboratory of Bioanalytical Chemistry, Graduate School of Pharmaceutical Sciences, The University of Tokyo, Tokyo, Japan ${ }^{3}$ Division of Molecular Biology, Institute for Genome Research, Institute of Advanced Medical Sciences, Tokushima University, Tokushima, Japan ${ }^{4}$ Laboratory of Cell Signaling, Graduate School of Pharmaceutical Sciences, The University of Tokyo, Tokyo, Japan ${ }^{5}$ Division of Immunology, Department of Infectious Diseases, Faculty of Medicine, University of Miyazaki, Miyazaki, Japan ${ }^{6}$ Genome Laboratory, Medical Research Institute, Tokyo Medical and Dental University (TMDU), Tokyo, Japan ${ }^{7}$ Department of Immunology, Graduate School of Medicine, University of the Ryukyus, Okinawa, Japan ${ }^{8}$ Department of Pathological Cell Biology, Medical Research Institute, TMDU, Tokyo, Japan ${ }^{9}$ Department of Clinical Proteomics, National Cancer Center Research Institute, Tokyo, Japan ${ }^{10}$ Division of Pediatrics, Faculty of Medicine, University of Miyazaki, Miyazaki, Japan
} 
BiP) from its luminal domain, followed by oligomerization and autophosphorylation. Activated PERK phosphorylates eukaryotic translation initiation factor 2 subunit $\alpha$ (eIF2 $\alpha$ ), leading to attenuation of global protein translation to reduce the ER load (Harding et al, 2000). Phosphorylation of elF2 $\alpha$ triggers the specific translation of ATF4, which activates the transcription of genes involved in the UPR. Although PERK enrichment in the mitochondria-associated ER membrane (MAM) has been reported to contribute to ER stress-induced apoptosis (Verfaillie et al, 2012; Rainbolt et al, 2013; Lebeau et al, 2018), whether PERK regulates mitochondrial homeostasis in BAs is largely unknown. Mitochondrial biogenesis is regulated by coordination between mitochondrial DNA transcription and nuclear DNA transcription activated by several transcription factors, including Nrf-1, Sp1, YY-1, ERRs, TFAM, and GA-binding protein transcription factor $\alpha$ subunit (GABP $\alpha$ ) (Dorn et al, 2015). The mechanisms by which these transcription factors strictly recognize mitochondrial conditions and are activated to regulate mitochondrial biogenesis are unclear.

Here, we show a novel function of PERK in BAs that is independent of the UPR. PERK is phosphorylated at the kinase insert region, presumably by a non-autophosphorylation mechanism, during BA differentiation. PERK is required for mitochondrial and thermogenic gene expression via transcriptional activation by GABP $\alpha$ and UCP1-mediated thermogenesis in vitro and in vivo. Overall, our data suggest that the activation of the PERK-GABP $\alpha$ pathway during BA differentiation is indispensable for mitochondrial inner membrane protein biogenesis and thermogenesis in BAT.

\section{Results}

\section{Development of mitochondria and increases in ER-mitochondria contact sites during BA differentiation}

The morphology of organelles, especially mitochondria, in BAT has been well characterized for more than half a century (Lever, 1957; Napolitano \& Fawcett, 1958). Recent reports have revealed that ERlocalized molecules contribute to mitochondrial biogenesis and function in BAT (Bartelt et al, 2018; Zeng et al, 2019). We first investigated the morphological changes in mitochondria and the ER in BAs during differentiation. Primary brown preadipocytes were isolated from the interscapular BAT (BBAT) of newborn mice and differentiated into BAs, and cells were harvested at each stage of differentiation (Fig 1A). A few LDs started to be observed on day 4, and multiple LDs emerged on day 6 (Fig S1), suggesting that adipogenesis was nearly completed on day 6 . The mitochondrial area and perimeter in differentiating and differentiated BAs (days 2, 4, and 6) were greater than those in brown preadipocytes (day 0) (Fig $1 \mathrm{~B}$ and $\mathrm{C}$ ). In contrast with mitochondria, the expanded ER areas and perimeters in brown preadipocytes (day 0) were substantially and significantly reduced during differentiation (Fig $1 \mathrm{~B}$ and D). OXPHOS-dependent ATP production was calculated by measurement of total and oligomycin A-insensitive intracellular ATP levels. During differentiation, the total amount of intracellular ATP was reduced (Fig 1E), whereas the ratio of OXPHOS-dependent ATP production to total ATP content was significantly increased (Fig 1F).
Moreover, we measured the oxygen consumption rate (OCR) and found that not only the basal OCR but also the extracellular acidification rate (ECAR), which represents glycolytic pathway activity, were increased during BA differentiation (Fig $1 \mathrm{G}$ and $\mathrm{H}$ ). However, because the rate of increase in basal OCR was higher than that of ECAR, the basal OCR/ECAR ratio was significantly higher in BAs than in brown preadipocytes (Fig 1I). These observations suggest that ATP production is dominantly mediated by the OXPHOS pathway in BAs. Consistent with these findings, the expression of OXPHOS complex members (respiratory chain complexes I, II, III, IV, and V) was clearly higher in BAs than in brown preadipocytes (Fig 1)). Moreover, not only the OXPHOS complexes, including cytochrome c oxidase subunit IV (COX4) but also other mitochondrial proteins, including UCP1, cytochrome c (Cyt C), and translocase of the outer membrane (Tom) 20, were markedly increased during differentiation (Fig 1K). Conversely, the amounts of the translocon component Sec61 $\alpha$ and the ER chaperone GRP78 were reduced (Fig 1K). Given all these findings, we conclude that BAs acquire developed mitochondria to enable ATP production mainly via activation of the OXPHOS pathway. Interestingly, the thinned ER was surrounded by mitochondria (Fig 1B, day 6). To quantify this finding, we measured contact sites, which were defined as sites at which the distance between the membranes of the two organelles was less than $30 \mathrm{~nm}$ (Fig 1B, yellow lines). The areas of ER-mitochondria contact sites, which were hardly detectable in brown preadipocytes (Fig 1B, day 0 ), were significantly increased during BA differentiation (Fig $1 \mathrm{~L}$ ). The ratios of ER-mitochondria contact sites to both the ER perimeter and the mitochondrial perimeter were significantly increased (Fig $1 \mathrm{M}$ and $\mathrm{N}$ ), suggesting that the ER and mitochondrial membranes may actively contact each other rather than meeting simply because of mitochondrial expansion. We, thus, hypothesize that ER-mitochondria crosstalk may play a role in the function of BAs.

\section{Phosphorylation of PERK during BA differentiation}

We next examined the involvement of the UPR signalling pathway in BAs. The amounts of PERK and IRE $1 \alpha$ decreased as BA differentiation proceeded because of the reduction in ER area (Fig S2). However, detailed time course experiments revealed that PERK band shifts were temporarily present after $6-12 \mathrm{~h}$ of culture with differentiation enhancement medium on day 2 (Fig 2A, top panel). These band shifts were eliminated by treatment with protein phosphatase (Fig 2B and C). Autophosphorylation of PERK was also detected with an antibody against phosphorylated PERK at Thr980 (Fig 2A, second panel). These findings suggest that the induction of BA differentiation triggers temporal phosphorylation, including autophosphorylation, of PERK on day 2. However, the electrophoresis pattern of IRE $1 \alpha$ was not affected during differentiation, and expression of the spliced form of XBP1, which is the result of activated IRE1 $\alpha$, was not observed (Fig $2 A$ and B). Because an antibody that specifically recognizes mouse ATF6 is not available, we examined the induction of GRP78, a downstream target of ATF6, and observed little changes in its expression during differentiation (Fig 2B). Our findings suggest that PERK is specifically phosphorylated among UPR sensors during BA differentiation. Under ER stress conditions, activated PERK phosphorylates elF2 $\alpha$, resulting in attenuation of global protein translation to reduce the ER protein load while promoting the specific translation of ATF4 (Harding et al, 

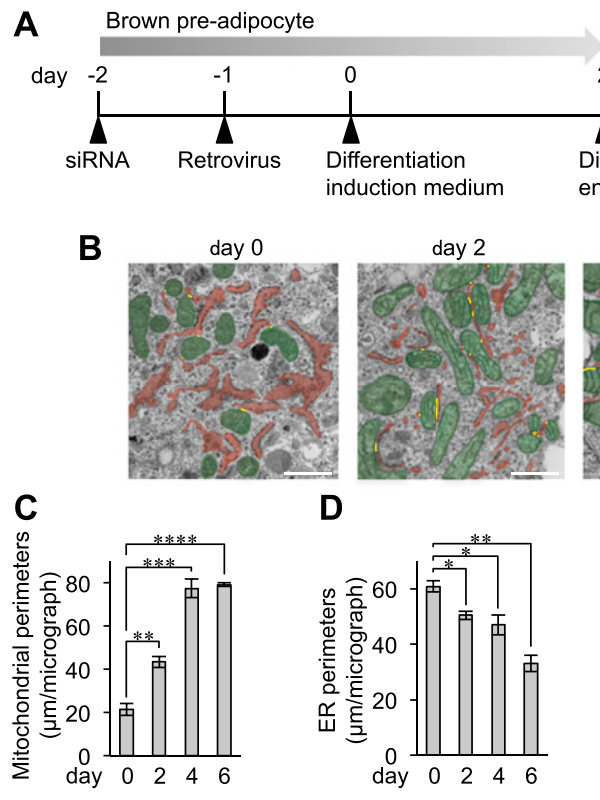

D

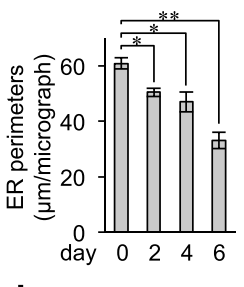

$\mathbf{J}$
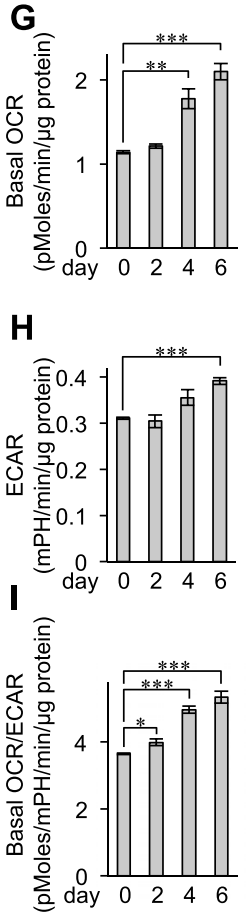

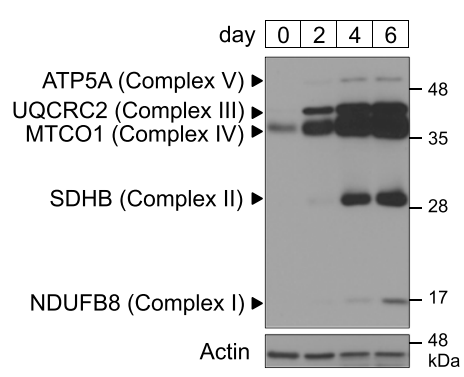

K

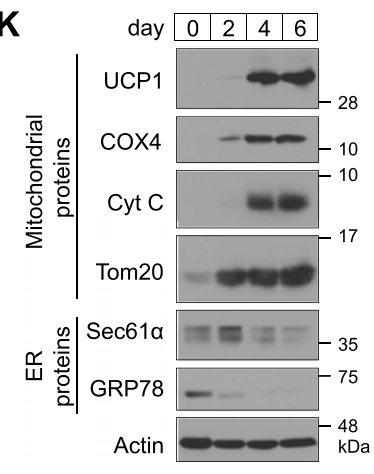

Brown adipocyte

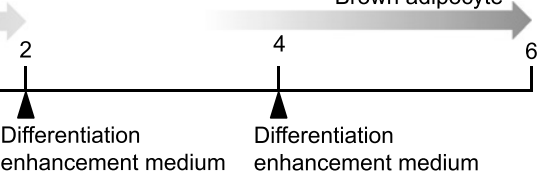

day 4

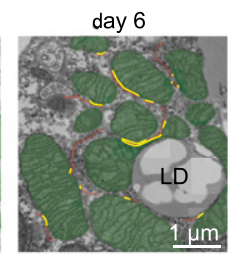

E

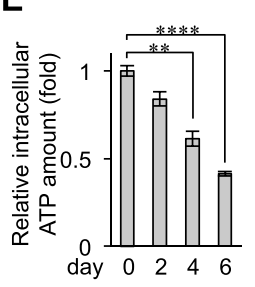

F

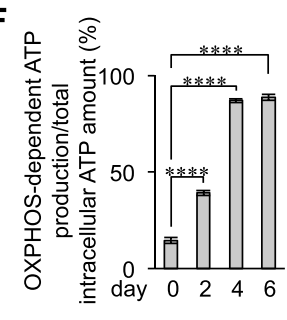

L

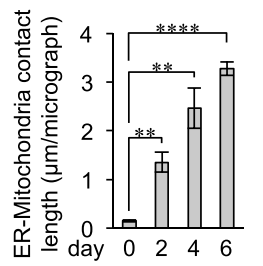

M

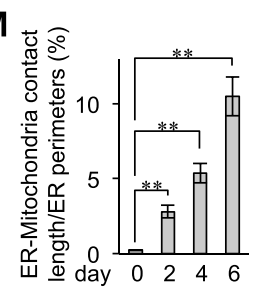

N

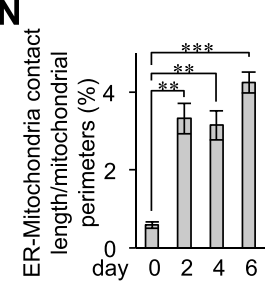

Figure 1. Morphological changes in the ER and mitochondria during brown adipocyte (BA) differentiation.

(A) Schematic representation of the BA preparation method used in this study. Primary brown preadipocytes were grown to confluence (day 2) and cultured with differentiation induction medium beginning on day 0 . After initiation of differentiation, the cell culture medium was changed to differentiation enhancement medium on day 2 and replaced with fresh medium on day 4. Cells on day 6 were used as differentiated BAs. siRNA transfection and retroviral transduction were performed on the indicated days. (B) Electron micrographs (EMs) of brown preadipocytes and BAs on day $0,2,4$, and 6 . Mitochondria and the ER have been false-coloured in green and red, respectively. The yellow lines denote ER-mitochondria contact sites (sites with $<30 \mathrm{~nm}$ of distance between two membranes). (C, D) Quantification of the ER (C) and mitochondrial (D) perimeters in EMs. Total length of the ER or mitochondrial perimeters in one EM was calculated with ImageJ software. Data are shown as the average from over $20 \mathrm{EMs}$ ( $n=3$ independent experiments). (E, F) Quantification of intracellular ATP content in BAs during differentiation. The cells were treated with or without $1 \mu \mathrm{g} / \mathrm{ml}$ oligomycin A for 45 min. (E, F) OXPHOS-dependent ATP production (F) was calculated from the total intracellular ATP content (E) and the oligomycin A-insensitive intracellular (glycolysis-dependent) ATP content. The data are shown as the percentage relative to total intracellular ATP content at each day ( $n=3$ independent experiments). (G, H, I) Measurement of basal oxygen consumption rate (OCR) (G) and extracellular acidification rate (ECAR) (H). (I) On day 0, 2, 4, and 6, the OCR and ECAR of brown preadipocytes and BAS were measured using a Seahorse analyser and XF Cel Mito stress test kit, and the basal OCR and basal OCR/ ECAR (I) were calculated as described in the Materials and Methods section. Data were normalized by total protein content ( $n=3$ independent experiments). (J, K) Expression levels of OXPHOS complexes (J) and mitochondrial and ER proteins $(K)$ during $B A$ differentiation. The cells were lysed on the indicated days and analysed by immunoblotting using the indicated antibodies. GPR94 and GRP78 protein was detected by a KDEL antibody. Actin was used as a loading control. (L, M, N) Quantification of

ER-mitochondria contact site in EMs. (L) Total length of contact site in one EM was calculated with ImageJ software (L). The data are shown as the average from 20 EMs ( $n=3$ independent experiments). (M, N) ER-mitochondria contact length was normalized to the ER (M) or mitochondrial ( $N$ ) perimeter and shown as percentage. Data information: data are presented as mean \pm SEM. ${ }^{\star} P<0.05,{ }^{* *} P<0.01,{ }^{* * *} P<0.001,{ }^{* * *} P<$ 0.0001 ( $t$ test). LD, lipid droplet.
1999; Vattem \& Wek, 2004). However, the levels of newly synthesized polypeptides, which were labeled with puromycin, were not reduced in differentiating cells (Fig 2D). The expression of the ATF4 protein and its target genes, CHOP and Gadd34, was also not increased (Fig 2E-G). Collectively, our results suggest that PERK may play a role via a pathway other than the elF2 $\alpha$-ATF4 axis in BA differentiation.

\section{Requirement of PERK for mitochondrial development in BAs}

To investigate the role of PERK in BAs, we first assessed the requirement of PERK for adipogenesis. The total amounts of LDs in
PERK-deficient cells were comparable with those in control cells (Fig 3A and B). Multiple LDs were observed not only in control siRNAtransfected (sictrl) cells but also in PERK-deficient BAs by electron microscopy (Fig 3C). Confocal microscopy revealed that the total areas of the ER and mitochondria in BAs were not affected by PERK deficiency (Fig S3A-C). Consistent with the results for the mitochondrial area, the amount of mitochondrial DNA relative to that of nuclear DNA did not differ between PERK-deficient BAs and control BAs (Fig S3D). Measurement of the organellar perimeter by electron microscopy revealed that the total ER perimeter was not affected by PERK deficiency, and a marginal but nonsignificant decrease in 
A

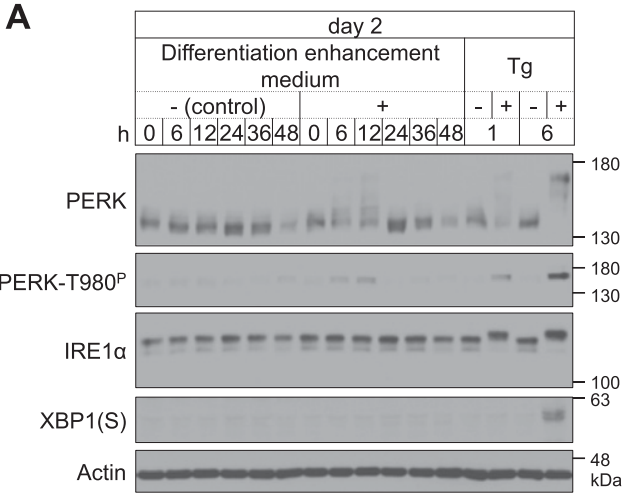

D

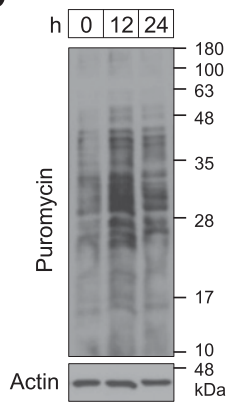

B

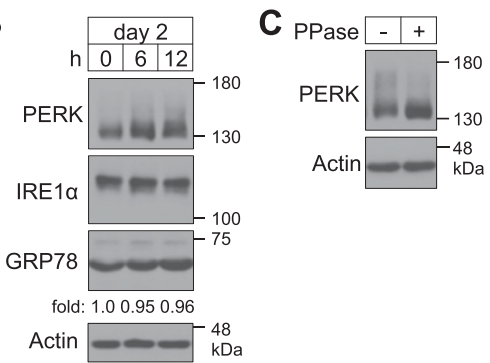

$\mathbf{F}$

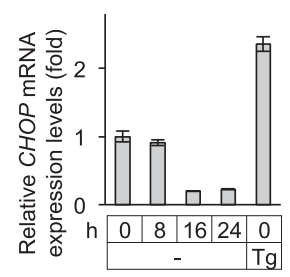

G

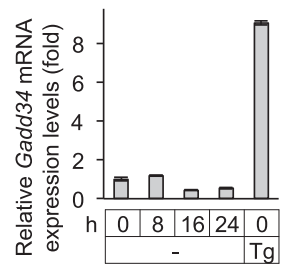

Figure 2. Phosphorylation of PERK during brown adipocyte differentiation.

$(A, B, C)$ Phosphorylation of PERK in differentiating cells. (A, B) Cells were cultured with differentiation medium ( $A$, - [control]) or differentiation

enhancement medium $(A,+$ and $B)$ for the indicated number of hours on day 2. (A) Cells treated with (+) or without (-) $40 \mathrm{nM}$ thapsigargin (Tg) for 1 or $6 \mathrm{~h}$ on day 2 were included as positive controls (A). (A, B) The cell lysates were analysed by immunoblotting (IB) with the indicated antibodies (A, B). (C) Lysates from cells cultured with differentiation enhancement medium for $12 \mathrm{~h}$ on day 2 were incubated with or without 2 units of $\lambda$ phosphatase (PPase) at $30^{\circ} \mathrm{C}$ for $30 \mathrm{~min}$ and analysed by IB with the indicated antibodies (C). (B) The expression of GRP78 was calculated and is shown as the ratio relative to actin expression (B). (D) Newly synthesized proteins in differentiating cells. Cells were incubated with $10 \mu \mathrm{g} / \mathrm{ml}$ puromycin for $10 \mathrm{~min}$ and lysed at the indicated time points on day 2. Newly synthesized proteins were detected by IB with an antipuromycin antibody. (E) IB analysis of ATF4 in differentiating cells. Cells were harvested after the indicated number of hours on day 2 and analysed by IB using the indicated antibodies. Cells treated with $40 \mathrm{nM}$ Tg for $6 \mathrm{~h}$ on day 2 were included as positive controls. $(\mathbf{F}, \mathbf{G})$ Expression of CHOP (F) or Gadd34 (G) mRNA in differentiating cells. Cells were harvested after the indicated number of hours on day 2, and total RNA was isolated. The data are shown as the fold change relative to the value at $0 \mathrm{~h}(n=3$ independent experiments). Cells treated with $40 \mathrm{nM}$ Tg for $12 \mathrm{~h}$ on day 2 were included as positive controls brown adipocytes. mitochondrial perimeter was observed in PERK-deficient BAs (Fig $\mathrm{S} 3 \mathrm{E}-\mathrm{G}$ ). The areas of the ER-mitochondria contact sites and the ratios of the contact sites to the ER or mitochondrial perimeters in PERK-deficient BAs were comparable with those in control BAs (Fig $\mathrm{S} 3 \mathrm{H}-\mathrm{J}$ ). Interestingly, electron microscopy revealed marked differences in the morphology of mitochondrial cristae among BAs (Fig 3D). The mitochondria in the control cells possessed dense parallel cristae, consistent with a previous report (Napolitano \& Fawcett, 1958), whereas significantly fewer mitochondria possessed dense parallel cristae in PERK-deficient BAs (Figs 3E and S3K). We next examined the expression levels of mitochondrial and ER proteins by immunoblotting (IB). Marginal increases in UPR-related ER protein levels were observed in PERK-deficient cells compared with control cells on day 0 (Fig 3F). The slight reduction in the outer membrane protein Tom20 expression on day 6 may have depended on the marginal reduction in mitochondrial perimeter (Figs $3 \mathrm{~F}$ and S3G). Drastic changes were also observed: the expression levels of mitochondrial inner membrane proteins (UCP1 and COX4) and a crista protein (Cyt C) were markedly reduced in PERK-deficient BAs (Fig 3F). Taken together, our findings suggest that PERK is required for crista formation and the expression of inner membrane and crista proteins during BA differentiation.

\section{Requirement of PERK for OXPHOS-dependent ATP production in BAs}

Next, to examine whether PERK deficiency affects mitochondrial functions, BAs were stained with a membrane potential-indicating dye (Fig $4 A$ ) and $\Delta \Psi m$ was quantified using a fluorescence-activated cell sorter (Fig 4B). The number of BAs exhibiting low $\Delta \Psi \mathrm{m}$ was significantly increased by PERK deficiency (Fig 4C). The total amount of intracellular ATP and the ratio of OXPHOS-dependent ATP production were reduced by PERK deficiency in BAs (Fig 4D and E). Moreover, the basal OCR, but not the ECAR, was significantly reduced in PERK-deficient BAs, resulting in a reduction in the basal OCR/ECAR ratio (Fig 4F-H). These phenotypes were attributable to the defective crista structure and the reduced expression of $\mathrm{COX}_{4}$ and Cyt $C$ in PERK-deficient BAs (Fig 3E and F). Collectively, these results strongly suggest that PERK contributes to OXPHOSdependent ATP production via regulation of crista formation and mitochondrial inner membrane biogenesis in BAs.

\section{Requirement of PERK phosphorylation for mitochondrial functions, but not for the UPR, in BAs}

In response to ER stress, activation of PERK is triggered by dissociation of GRP78 from its ER luminal domain, after which PERK is oligomerized and autophosphorylated (Bertolotti et al, 2000). To examine whether the phosphorylation of PERK during BA differentiation is caused by the ER stress, we created a retrovirusencoded PERK mutant with deletion of the ER luminal domain (PERK- $\triangle L D$ ), which does not respond to ER stress and used it to infect PERK-deficient BAs (Figs 5A and S4A). The deletion of the ER luminal domain had no effect on the phosphorylation of PERK (Fig $5 B$, top panel, parentheses). In addition, a band shift of a catalytically inactive PERK mutant (PERK- $L \mathrm{LD}-\mathrm{KA}$ ) that represents the 


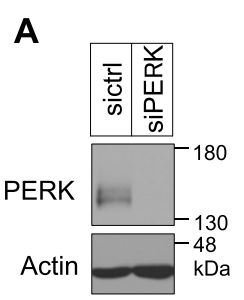

C
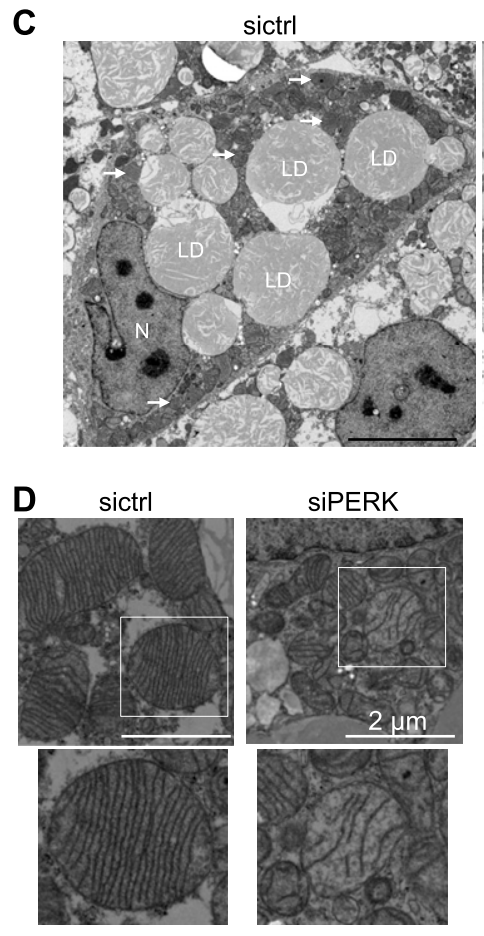

E

$\square$ Dense parallel crista morphology
$\square$ Sparse crista morphology

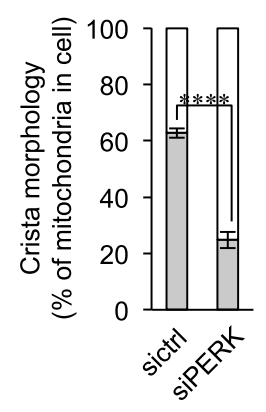

B
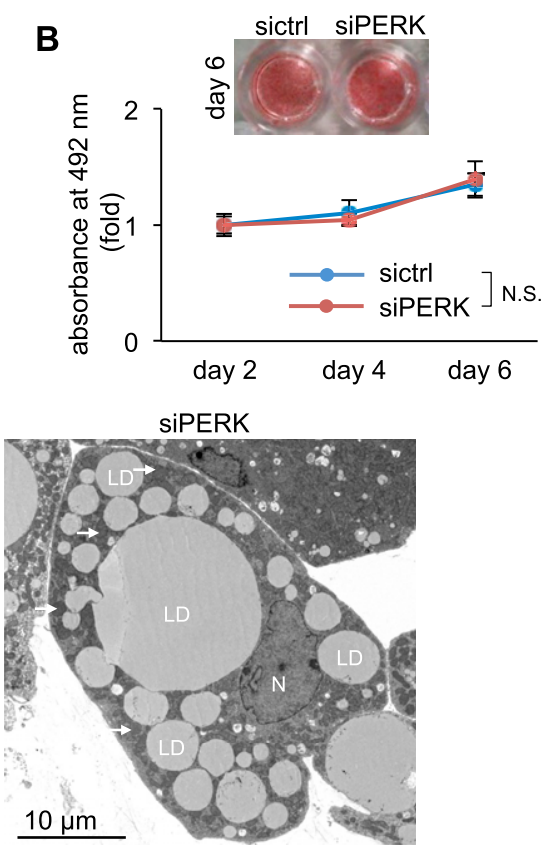

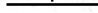

Figure 3. Requirement of PERK for mitochondrial development in brown adipocytes (BAs).

(A) Knockdown efficiency of PERK in BAs. siRNAtransfected cells were lysed on day 6 and analysed by immunoblotting with an anti-PERK antibody. (B) PERK-independent adipogenesis. siRNA-transfected BAs were stained with oil red O (images), and the isolated oil red $\mathrm{O}$ from cells was measured by quantification of light absorbance at $492 \mathrm{~nm}$ (graph). Data are shown as the fold change relative to the value on day 2 (graph) ( $n=3$ independent experiments). (c, D, E) Requirement of PERK for dense parallel cristae formation in mitochondria. (C, D, E) Brown preadipocytes were transfected with sictrl or SiPERK, fixed on day 6, and analysed by electron microscopy (arrows, mitochondrion; LD, lipid droplet; $\mathrm{N}$, nucleus) (C D, E). Mitochondria were divided into mitochondria possessing dense parallel cristae and mitochondria possessing sparse cristae and were counted (in a total of 50 individual cells) (E). The white square denotes the magnified region ( $n=3$ independent experiments). (F) Requirement of PERK for mitochondrial (inner membrane and crista) and ER proteins. Brown preadipocytes were transfected with siRNAs. The lysates were analysed by immunoblotting with the indicated antibodies. GPR94 and GRP78 protein was detected by a KDEL antibody. (B, E) Data information: data are presented as mean \pm SEM. NS; $* \star \star \star * P<0.0001$ (repeated measures ANOVA in (B), $t$ test in (E)). phosphorylated form was observed in differentiating PERK-deficient cells (Fig 5C). Considering the observation of PERK autophosphorylation (Fig 2A), our findings suggest that PERK is phosphorylated, presumably by a non-autophosphorylation mechanism in addition to autophosphorylation, during BA differentiation independently of the ER stress. We next investigated the phosphorylated amino acid residues of PERK in differentiating cells. Liquid chromatography coupled with tandem mass spectrometry (LC-MS/MS)-based phosphoproteomic analysis was performed using immunopurified Flag-tagged PERK- $\triangle L D-K A$. Ser719 in the kinase insert region was revealed to be a strong candidate amino acid residue for phosphorylation during BA differentiation (Figs S4B and 5A). Database analysis using PhosphoSitePlus (https://www.phosphosite.org/homeAction) suggested that Ser715 might also be phosphorylated. Because the set of Ser715, Ser717 
A

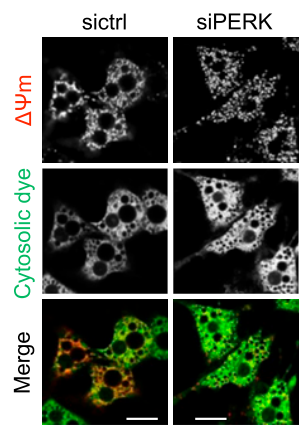

B

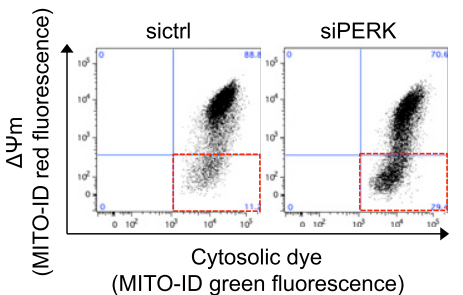

C

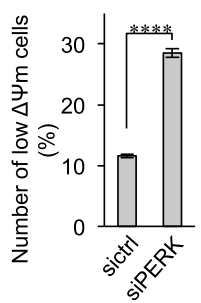

D
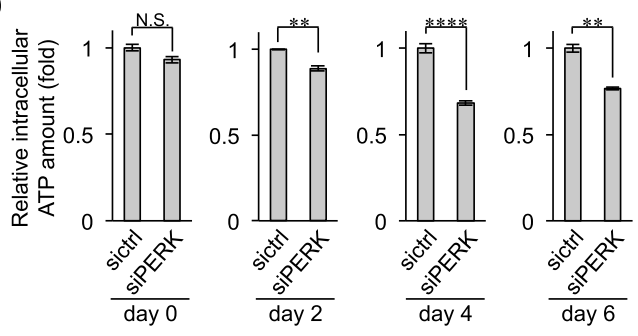

E
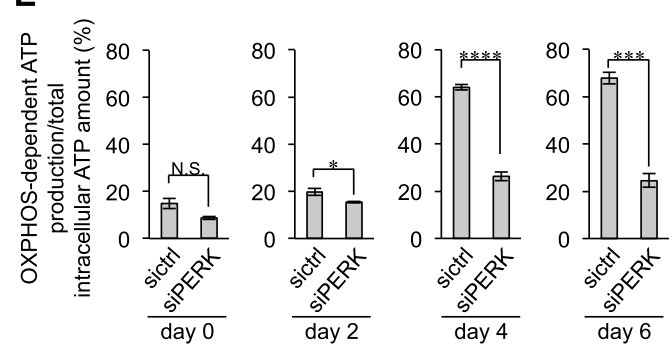

$\mathbf{F}$

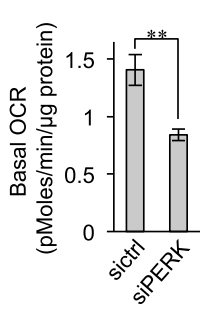

G

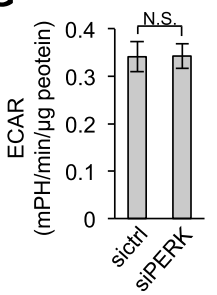

$\mathrm{H}$ 토

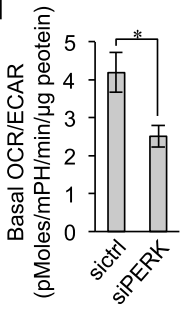

Figure 4. Requirement of PERK for $\Delta \Psi \mathrm{m}$ and OXPHOSdependent ATP production in brown adipocytes (BAs).

(A) Fluorescence images of $\Delta \Psi \mathrm{m}$ in BAs. siRNAtransfected BAs were treated with MITO-ID for $15 \mathrm{~min}$ at room temperature on day 6 . The $\Delta \psi \mathrm{m}$ was visualized by confocal fluorescence microscopy. The intensity of red fluorescence denotes the $\Delta \Psi \mathrm{m}$. Green

fluorescence denotes cytosolic dye, which was used as the loading control. Scale bar, $5 \mu \mathrm{m}$. (B, C) Requirement of PERK for maintenance of $\triangle \Psi \mathrm{m}$. siRNA-transfected BAs were stained with MITO-ID and necrosis detection reagents for $15 \mathrm{~min}$ at room temperature on day 6. (B) Necrotic cells were removed, and the population of cells showing low $\Delta \Psi \mathrm{m}$ was analysed by flow cytometry (B, more than 10,000 cells for each sample). (C) The cell population with low $\Delta \Psi_{m}$ was surrounded with the dotted red square and counted (C). Data are shown as the percentages relative to the total number of cells ( $n=$ 3 independent experiments). (D, E) Requirement of PERK for OXPHOS-dependent ATP production. (D, E) sictrl- or siPERK-transfected cells were harvested on the indicated days, and the total intracellular ATP content (D) and OXPHOS-dependent ATP production (E) were measured as described in Fig $1 E$ and $F(n=3$ independent experiments). (F, G, H) Requirement of PERK for basal oxygen consumption rate (OCR) in BAs. The OCR and extracellular acidification rate of sictrl- or siPERK-transfected cells were measured using a Seahorse analyser and XF Cell Mito stress test kit. (F, G, H) Data were normalized by total protein content and show the basal OCR (F), extracellular acidification rate $(\mathrm{G})$, and basal OCR/ECRA $(\mathrm{H})(n=3$ independent experiments). Data information: data are presented as mean \pm SEM. NS; ${ }^{*} P<0.05 ;{ }^{* *} P<0.01 ;{ }^{* *} P<0.001 ;{ }^{* * * *} P$ $<0.0001$ ( $t$ test). and Ser719 (3S) is conserved in rodents and humans (Fig 5D), a polyclonal antibody against a peptide containing phosphorylated 3S (PSPERS ${ }^{P}$ RS $^{P}$ FS $^{P}$ VGI) was raised in rabbits and was designated as an antibody against phosphorylated PERK at Ser715, Ser717, and/or

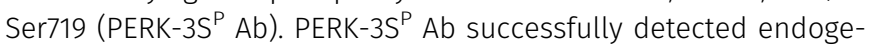
nous PERK and exogenous PERK- $\triangle$ LD, but not PERK- $\triangle$ LD-3SA (in which serine was substituted with alanine at Ser715, Ser717, and Ser719), during BA differentiation (Fig 5B, second panel). Our results using PERK-3S ${ }^{P}$ Ab provide evidence that PERK is physiologically phosphorylated in the kinase insert region, at least at Ser719, during differentiation.

We next investigated the requirements of Ser715, Ser717, and Ser719 for mitochondrial function. The reduced expression of UCP1, COX4, and Cyt $\mathrm{C}$ and the reduced $\triangle \Psi \mathrm{m}$ and OXPHOS-dependent ATP production caused by PERK deficiency were ameliorated in cells transfected with PERK- $\triangle$ LD but not in those transfected with PERK$\triangle$ LD-KA or PERK- $\triangle \mathrm{LD}-3 \mathrm{SA}$ (Fig $5 \mathrm{E}-\mathrm{G}$ ), suggesting that both kinase activity and phosphorylation at Ser715, Ser717, and / or Ser719 may be required for PERK-mediated mitochondrial inner membrane protein biogenesis and functions in BAs. Although PERK was also recognized by PERK-3S $\mathrm{Ab}$ in thapsigargin- or tunicamycin-treated cells (Fig S4C), attenuation of ER stress-induced transcriptional activation by ATF4, which was measured using an amino acid response element-luciferase reporter (AARE-luc) (Miyake et al, 2016), in PERK-deficient BAs was completely eliminated by exogenously expressed PERK-3SA, as it was by wild-type (WT) PERK (Fig 5H). Collectively, our observations suggest that the kinase activity and
Ser715, Ser717, and / or Ser719 residues of PERK are indispensable for mitochondrial functions but not for the UPR.

\section{Involvement of GABP $\alpha$ in PERK-mediated mitochondrial inner membrane biogenesis}

To examine whether PERK regulates the transcription of BA-related genes, total RNA harvested from control and PERK-deficient BAs on day 4 was analysed using quantitative reverse transcriptase-PCR (qPCR). PERK deficiency significantly reduced the mRNA levels of Cox4i1, Cox8b, Cox7a1, and Cycs, which are the genes associated with OXPHOS complexes, and UcP1, Cidea, and Dio2, which are the genes related to BA function (Fig 6A). PERK was also required for the expression of Ppargc1a mRNA, which encodes the transcriptional coactivator peroxisome proliferator-activated receptor $y$ coactivator$1 \alpha($ PGC- $1 \alpha)$ that regulates OXPHOS complex genes and Ucp1, but not for the expression of Ppargc1b mRNA (Fig 6A). In contrast, the mRNA expression of Pparg and Prdm16, which are the master regulator genes of BA differentiation, was not changed by PERK deficiency (Fig 6A). These results suggest that PERK specifically regulates the transcriptional expression of subsets of mitochondria-related genes. To understand the mechanism by which PERK regulates gene expression, we performed RNA sequencing analysis of control and PERK-deficient cells after 16 or $24 \mathrm{~h}$ of culture with differentiation enhancement medium on day 2 . PERK deficiency reduced the expression levels of 381 genes at both time points (by $>1.32$-fold) (Fig $6 \mathrm{~B}$ and Table S1). We next analysed the conserved transcription 

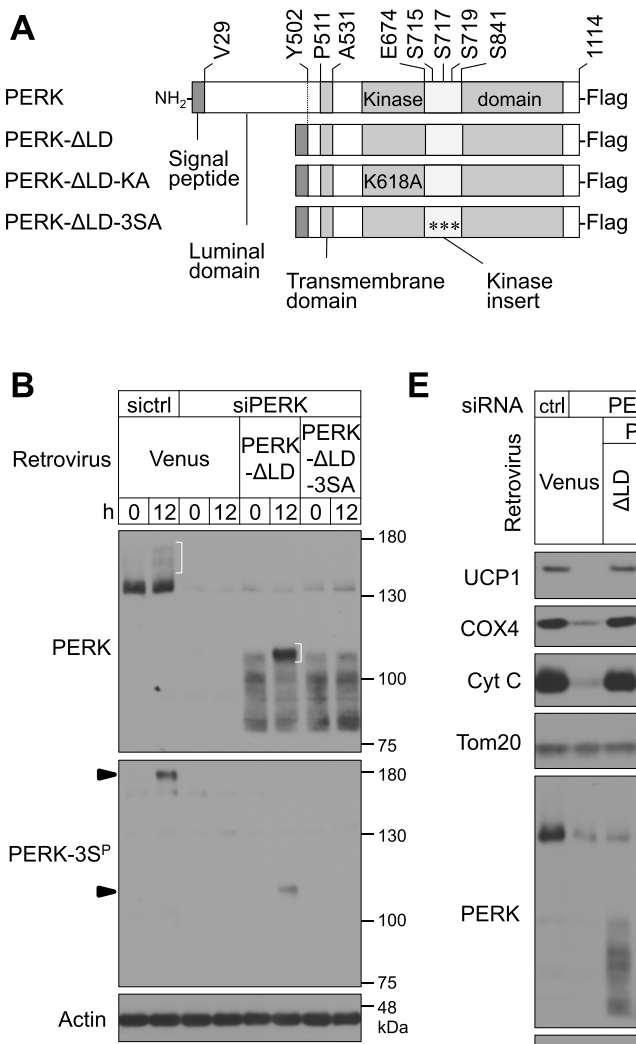

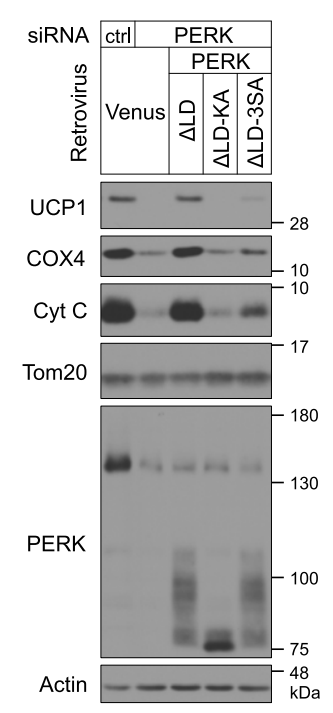

D

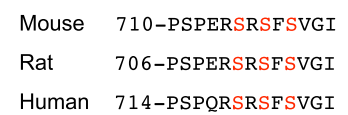

G

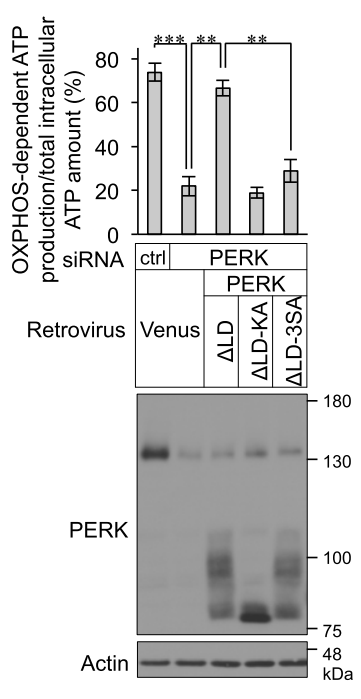

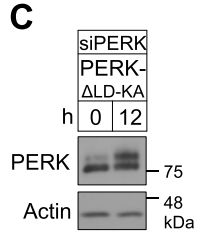

$\mathbf{F}$

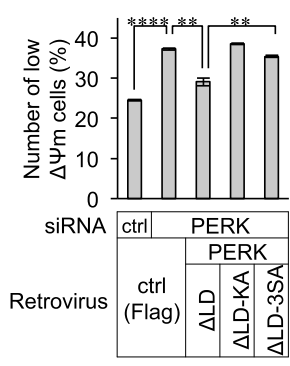

H

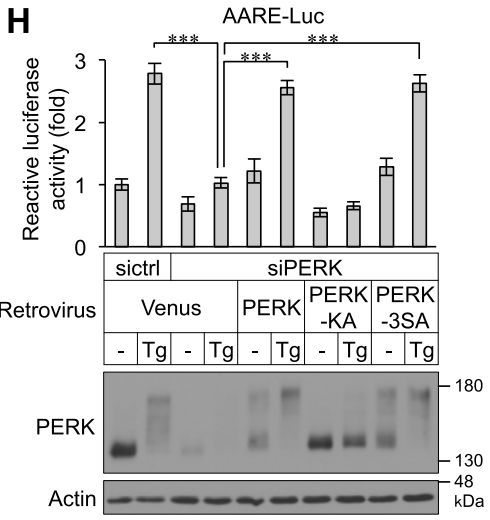

Figure 5. Requirement of PERK-3S phosphorylation for mitochondrial functions, but not for the unfolded protein response, in brown adipocytes (BAs).

(A) Schematic representation of the C-terminal Flagtagged full-length PERK, luminal domain-deleted ( $\triangle \mathrm{LD}$; $\Delta$ Val29-Tyr502) mutant PERK, kinase-negative $\triangle \mathrm{LD}(\Delta \mathrm{LD}-\mathrm{KA}$; substitution of lysine to alanine at Lys618) mutant PERK, and $3 S A \triangle L D(\triangle L D-3 S A$; substitution of serine to alanine at Ser715, Ser717, and Ser719) mutant PERK. (B) Immunoblotting (IB) analysis of the phosphorylation of endogenous or exogenously expressed PERK in differentiating cells. siRNA-transfected cells were infected with retroviruses expressing Venus, PERK- $\Delta L D$, or PERK$\triangle$ LD-3SA and cultured with differentiation enhancement medium for the indicated number of hours on day 2. The cell lysates were analysed by IB with anti-PERK and anti-PERK- $3 S^{\mathrm{P}}$ antibodies. Actin was used as a loading control. Arrowheads indicate the phosphorylated PERK at Ser715, Ser717, and/or Ser719. White parentheses indicate the phosphorylated PERK during BA differentiation. (C) IB analysis of exogenously expressed PERK- $\triangle L D-K A$ in differentiating cells. SiPERKtransfected cells were infected with retroviruses expressing PERK- $\triangle L D-K A$. The cells were lysed after the indicated number of hours on day 2 and analysed by IB with the indicated antibodies. (D) Alignment of the backbone peptide of PERK for the generation of phosphoSer715, phospho-Ser717, and phospho-Ser719 antibodies from the indicated various species. Amino acids conserved with Ser715, Ser717, and Ser719 in mouse PERK are highlighted in red. (E) Requirement of PERK kinase activity and phosphorylation at Ser715, Ser717, and/or Ser719 for the expression of mitochondrial inner membrane and crista proteins. siRNA-transfected cells were infected with retroviruses expressing Venus, PERK$\triangle L D$, PERK- $\Delta L D-K A$, or PERK- $L L D-3 S A$, lysed on day 6 and analysed by IB with the indicated antibodies. (F) Requirement of PERK kinase activity and phosphorylation at Ser715, Ser717, and/or Ser719 for maintenance of $\Delta \Psi \mathrm{m}$ in BAs. siRNA-transfected primary brown preadipocytes were infected with the indicated retroviruses. The $\Delta \Psi \mathrm{m}$ values were analysed by flow cytometry (more than 10,000 cells for each sample) and are shown as described in Fig 4C ( $n=3$ independent experiments). (G) Requirement of PERK kinase activity and phosphorylation at Ser715, Ser717, and/or Ser719 for OXPHOS-dependent ATP production. siRNA-transfected primary brown preadipocytes were infected with the indicated retroviruses. The ATP levels were measured (top) and are shown as described in Fig 1F. The cell lysates were analysed by IB with the indicated antibodies (bottom) $(n=$ 3 independent experiments). (H) Role of PERK

phosphorylation at Ser715, Ser717, and/or Ser719 on the elF2 $\alpha$-ATF4 pathway in differentiating cells. siRNA-transfected brown preadipocytes were co-transfected with $10 \mu \mathrm{g}$ of AARE-luc and $1 \mu \mathrm{g}$ of Renilla-luc and infected with the indicated retroviruses expressing Venus, PERK, PERK-KA, or PERK-3SA. The cells were stimulated with $40 \mathrm{nM}$ Tg for $12 \mathrm{~h}$ on day 2, and the relative luc activity was measured (top). AARE-luc activity was normalized to Renilla-luc activity. Data are shown as the fold change relative to the value in nonstimulated sictrl-transfected BAs. The cell lysates were analysed by IB with the indicated antibodies (bottom) $(n=3$ independent experiments). Data information: data are presented as mean \pm SEM. ${ }^{* *} P<0.01,{ }^{* \star *} P<0.001,{ }^{* \star * *} P<0.0001$ ( $t$ test $)$.

factor-binding motifs of these genomic DNA sequences. The binding motifs of transcription factor EB, CAMP response element-binding protein (CREB) and GABP $\alpha$ were enriched in 381 genes regulated by PERK in differentiating cells (Fig 6C). Similar to the case in differentiated BAs, phosphorylated PERK was detected by PERK-3S ${ }^{P} A b$ in HEK293 cells habituated to OXPHOS through culture with no-glucose medium (Fig S5A and B). RNA sequencing analysis of HEK293 cells revealed that the expression levels of 630 genes were lower in PERK$\mathrm{KO}$ cells than in WT cells (by >1.49-fold) (Table S2). Among them, 111 genes were categorized as mitochondria-related genes by gene annotation enrichment analysis (Fig S5C), and the binding motif of GABP $\alpha$ was found to be enriched in 48 mitochondria-related genes in HEK293 cells (Fig S5D). GABPa regulates the transcription of OXPHOS complex genes, including Cycs and Cox4i1, and functions as a regulator of glycolytic beige adipocyte differentiation (Chen et al, 2009, 2019). Taken together, the results obtained from BAs and HEK293 cells as well as those from previous reports suggest that PERK may activate the GABP $\alpha$ pathway, resulting in mitochondrial inner membrane biogenesis in BAs. To investigate this hypothesis, we examined whether PERK is required for transcriptional activation by GABP $\alpha$ in 
A
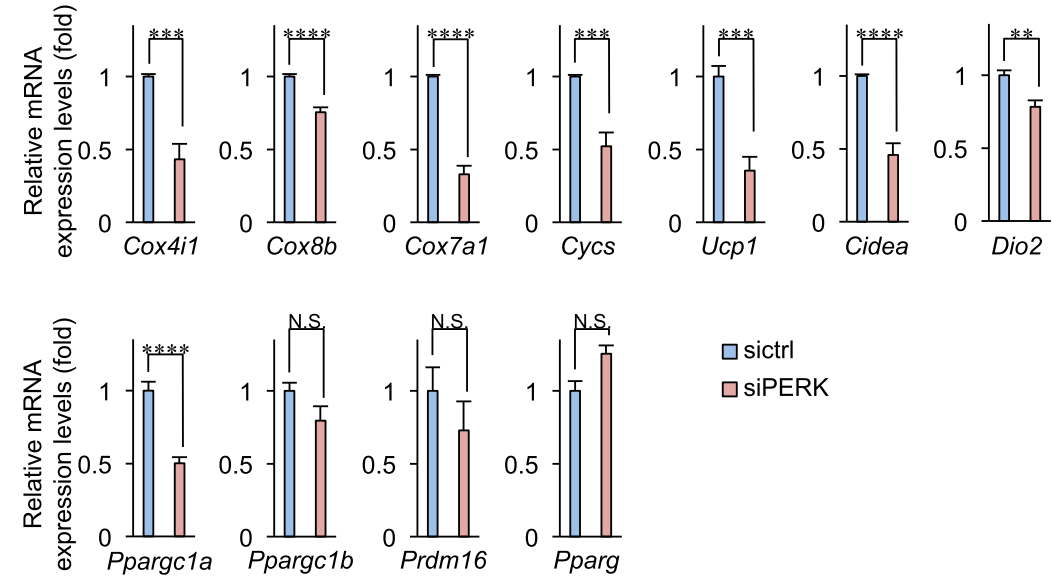

$\square$ sictrl

$\square$ SIPERK

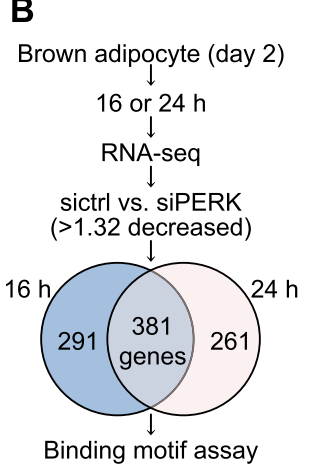

C

\begin{tabular}{|c|c|c|c|}
\hline $\begin{array}{l}\text { Transcription } \\
\text { factor }\end{array}$ & & Sequence & $P$-value \\
\hline TFEB & $\begin{array}{c}\text { Known motif } \\
\text { De Novo }\end{array}$ & $\begin{array}{r}\text { TGAc TCA } \\
A C T \subset A C\end{array}$ & $8.51 \times 10^{-5}$ \\
\hline CREB & $\begin{array}{c}\text { Known motif } \\
\text { De Novo }\end{array}$ & $\begin{array}{l}\text { CAC } T_{A C} \\
\text { ACGTAC }\end{array}$ & $2.75 \times 10^{-4}$ \\
\hline GABP $\alpha$ & $\begin{array}{c}\text { Known motif } \\
\text { De Novo }\end{array}$ & $\begin{array}{l}c C G A A A \\
A C G A A C\end{array}$ & $1.4 \times 10^{-3}$ \\
\hline
\end{tabular}

Figure 6. Mitochondrial gene expression in PERKdeficient brown adipocytes.

(A) qPCR analysis of brown adipocyte-related genes in differentiating cells. Total RNA from siRNA-transfected cells was isolated on day 4 . The gene expression levels were analysed by qPCR. The mRNA expression of the indicated genes was normalized to that of S18. Data are shown as the fold change relative to the value in sictrl-transfected cells ( $n=3$ independent experiments). (B) Venn diagram of common genes between two time points (sictrl versus siPERK, $>1.32-$ fold decrease). sictrl- or siPERK-transfected differentiating cells were cultured with differentiation enhancement medium for 16 or $24 \mathrm{~h}$, and mRNA was isolated. RNA sequencing data were analysed with a Venn diagram. (C) Motif enrichment analysis of PERKrelated genes in differentiating cells. Data information: data are presented as mean \pm SEM. NS; ${ }^{* *} P$ $<0.01 ;{ }^{* * *} P<0.001 ;{ }^{* * * *} P<0.0001$ ( $t$ test). differentiating cells with a luciferase assay using the promoter region of mitochondrial translation initiation factor 2 containing the binding site for GABP $\alpha$ (Hayashi et al, 2007). The expression level of GABP $\alpha$ protein was not affected by PERK deficiency (Fig S5E); however, transcriptional activation by $\mathrm{GABP} \alpha$ was significantly reduced in PERK-deficient BAs, whereas it was not reduced in IRE1 $\alpha$ - or ATF6deficient cells (Fig 7A and B). This effect was attenuated by exogenously expressed PERK- $\triangle$ LD but not PERK- $\triangle$ LD-KA or PERK- $\triangle L D-3 S A$ (Fig 7C), suggesting that PERK specifically regulates the GABP $\alpha$ pathway as a result of PERK kinase activation and phosphorylation at Ser715, Ser717, and/or Ser719 during BA differentiation. We next examined whether the PERK-GABP $\alpha$ axis regulates mitochondrial inner membrane protein biogenesis and function in BAs. The reduced expression of UCP1, COX4, and Cyt C and inhibition of OXPHOSdependent ATP production in PERK-deficient BAs were ameliorated by coexpression of $\mathrm{GABP} \alpha$ with GA-binding protein subunit $\beta-1$ (GABP $\beta 1$ ), which is a coactivator of GABP $\alpha$ (Fig 7D, lanes 2 and 4). UCP1, COX4, and Cyt $\mathrm{C}$ expression and OXPHOS-dependent ATP production were reduced by GABP $\alpha$ deficiency just as they were by PERK deficiency (Fig S5F). PERK- $\triangle \mathrm{LD}$ did not reconstitute the phenotype in GABP $\alpha$-deficient BAs (Fig 7D, lanes 5 and 6), suggesting that GABP $\alpha$ functions downstream of PERK in mitochondrial inner membrane biogenesis and function.

\section{Requirement of PERK for thermogenesis of BAs}

One of the most important roles of mitochondria in BAs is thermogenesis. To clarify the role of PERK in intracellular thermogenesis, we performed a thermogenic analysis using a fluorescent polymeric thermometer in single BAs (Gota et al, 2009). Treatment of differentiated BAs with the $\beta_{3}$-adrenoceptor $\left(\beta_{3} A R\right)$ agonist CL316,243 increased the intracellular temperature in control BAs but not in PERK-deficient BAs (Fig 8A). The percentage of retrovirus-infected BAs expressing the GFP-variant Venus was $~ 67 \%$ (data not shown). At this infection efficiency, the thermogenic defect in PERK-deficient BAs was significantly improved by exogenously expressed PERK- $\triangle$ LD (Fig 8A). However, PERK- $\triangle$ LD-3SA had no effect on the phenotype (Fig $8 A)$. Considering the result that PERK- $\triangle L D-K A$ did not rescue the reduced expression of UCP1 in PERK-deficient BAs at all (Fig 5E), PERK kinase activation and phosphorylation at Ser715, Ser717, and/or Ser719 may be indispensable for thermogenesis.

Finally, we examined the requirement of PERK for thermogenesis in vivo. In newborn mice, iBAT thermogenesis is essential for body temperature maintenance (Liu et al, 2003). We analysed iBAT derived from newborn mice within $12 \mathrm{~h}$ after birth to avoid the effect of hyperglycaemia due to progressive degeneration of pancreatic secretory cells in $P E R K^{-/-}$mice (Zhang et al, 2002). Although there were no obvious differences in gross and histological observations (Fig S6A and B), electron microscopic analysis revealed that the numbers of mitochondria with dense parallel cristae were significantly decreased in iBAT derived from $P E R K^{-/-}$mice compared with that derived from WT mice (Fig 8B). In accord with the results of the in vitro experiments, the expression levels of UCP1, COX4, and Cyt C, but not Tom20, were significantly reduced in iBAT derived from PERK ${ }^{-1-}$ mice (Fig $8 \mathrm{C}-\mathrm{G}$ ). The temperature of the skin overlying iBAT in newborn mice reflects the extent of iBAT thermogenesis (Hodges 
A

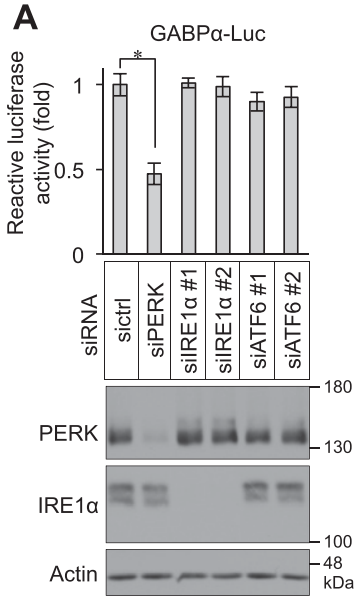

B

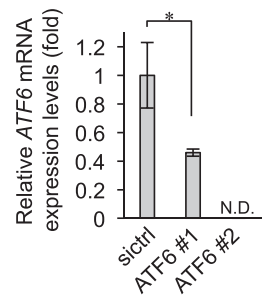

C

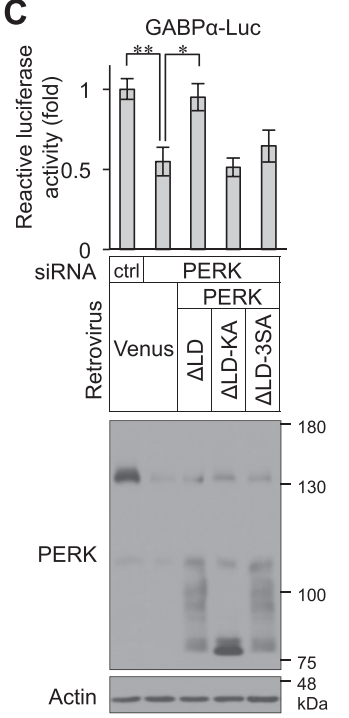

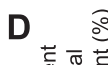

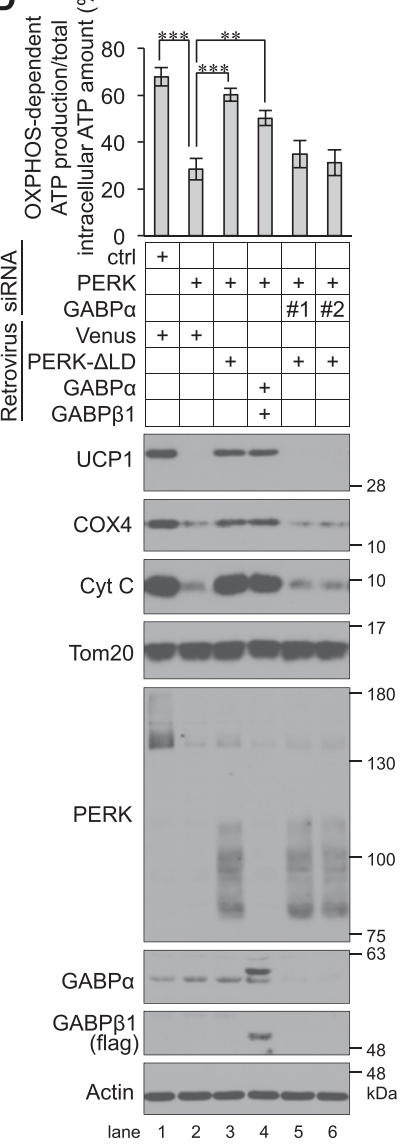

Figure 7. Involvement of GABP $\alpha$ in PERK-mediated mitochondrial inner membrane protein biogenesis.

(A) Role of inositol-requiring enzyme $1 \alpha$ or ATF6 in the transcriptional activity of GABP $\alpha$ in differentiating cells. siRNA-transfected brown preadipocytes were cotransfected with GABP $\alpha$-luc and Renilla-luc. Relative luc activity was measured on day 3 (top). GABP $\alpha$-luc activity was normalized to Renilla-luc activity. Data are shown as the fold change relative to the value in sictrl-transfected brown adipocytes (BAs) $(n=3$ independent experiments). The knockdown efficiency of siPERK and siRNAs against inositolrequiring enzyme $1 \alpha$ (silRE1 $\alpha \# 1$ and $\operatorname{silRE} 1 \alpha \# 2$ ) was analysed by immunoblotting (IB) (bottom). (B) Knockdown efficiency of ATF6 siRNA (siATF6) transfection. Primary brown preadipocytes were transfected with sictrl or with ATF6 \#1 or ATF6 \#2 SiRNA. The amount of ATF6 mRNA on day 3 was analysed by qPCR and normalized to that of S18 mRNA. The data are shown as the fold change relative to the value in sictrl-transfected BAs ( $n=3$ independent experiments). (c) Requirement of PERK for the transcriptional activity of GABP $\alpha$ in differentiating cells. siRNA-transfected brown preadipocytes were cotransfected with $10 \mu \mathrm{g}$ of GABP $\alpha$-luc and $1 \mu \mathrm{g}$ of Renillaluc and infected with retroviruses expressing Venus, PERK- $\triangle L D$, PERK- $\triangle L D-K A$, or PERK- $\triangle L D-3 S A$. The relative luc activity was measured on day 3 (top). GABP $\alpha$-luc activity was normalized to Renilla-luc activity. Data are shown as the fold change relative to the value in sictrl-transfected BAs ( $n=3$ independent experiments) The cell lysates were analysed by IB with the indicated antibodies (bottom). (D) Requirement of the PERK-GABP $\alpha$ pathway for the expression of mitochondrial proteins and OXPHOS-dependent ATP production. ATP levels were measured (top) and are shown as described in Fig 1F. Data are shown as the percentage relative to total intracellular ATP content in each transfected BA $(n=4$ independent

experiments). siRNA-transfected cells were infected with the indicated retroviruses, lysed on day 6 , and analysed by IB with the indicated antibodies (bottom). Data information: data are presented as mean \pm SEM. ${ }^{*} P<0.05,{ }^{* *} P<0.01,{ }^{* * *} P<0.001$ ( $t$ test). N.D., not detectable. et al, 2008). iBAT thermogenesis was, thus, measured by using an infrared thermographic imaging system. During exposure to $16^{\circ} \mathrm{C}$, newborn mice experienced cold stress-induced hypothermia, which was exacerbated in PERK ${ }^{--}$mice $(P=0.01037)$ (Fig 8H). Collectively, our observations strongly suggest that body temperature is regulated by BAT-derived thermogenesis through PERK-mediated mitochondrial inner membrane protein biogenesis in mice exposed to cold stress.

\section{Discussion}

In this work, we provide evidence that the ER-resident kinase PERK is essential for mitochondrial development in BAs. PERK is phosphorylated at Ser715, Ser717, and/or Ser719 by a mechanism that is independent of ER stress during BA differentiation. PERK kinase activity and its phosphorylation at Ser715, Ser717, and/or Ser719 are required for the expression of mitochondrial inner membrane and crista proteins, including the OXPHOS complexes and UCP1, as a result of transcriptional activation by GABP $\alpha$. We also propose a novel role in which PERK aids in OXPHOS-dependent ATP production and UCP1-mediated thermogenesis in BAs. PERK has been reported to regulate mitochondrial functions and homeostasis through the conventional PERK-eIF2 $\alpha$-ATF4 axis in cells other than BAs (Hori et al, 2002; Bouman et al, 2011; Rainbolt et al, 2013; Lebeau et al, 2018). A recent study has shown that the PERK-ATF4 axis increases the expression of supercomplex assembly factor 1 , which promotes the formation of mitochondrial respiratory supercomplexes under ER and nutrient stress conditions (Balsa et al, 2019). However, increased expression of ATF4 and induction of downstream target genes of ATF 4 were not observed on day 2 (Fig 2E-G). Thus, we conclude that mitochondrial inner membrane protein biogenesis in BAs is regulated by a novel PERK-GABP $\alpha$ axis. However, several questions regarding the upstream and downstream targets of this mechanism must be answered by further investigations. One important uncertainty is the location at which PERK recognizes mitochondrial conditions during BA differentiation. To address this uncertainty, it will be necessary to visualize the sites at which PERK is phosphorylated by the unidentified kinase. The polyclonal PERK$3 S^{P} A b$ used in this manuscript is useful for the detection of phosphorylated PERK by IB but not by immunocytochemistry. Successful generation of another PERK-3S $A b$ that is useful for immunocytochemistry and/or immunoelectron microscopy would enable investigation of this issue. The mediator that conveys the alterations in mitochondrial conditions to PERK or its upstream kinase also remains to be identified. In BAs treated with the 
A

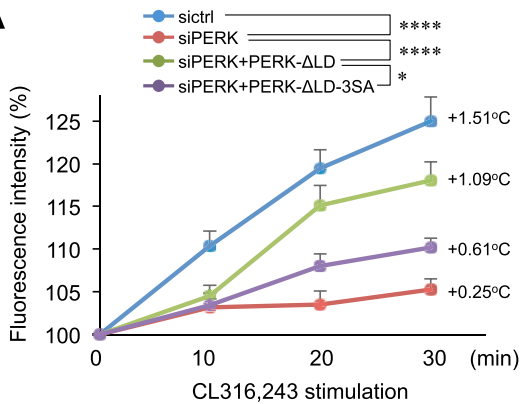

C
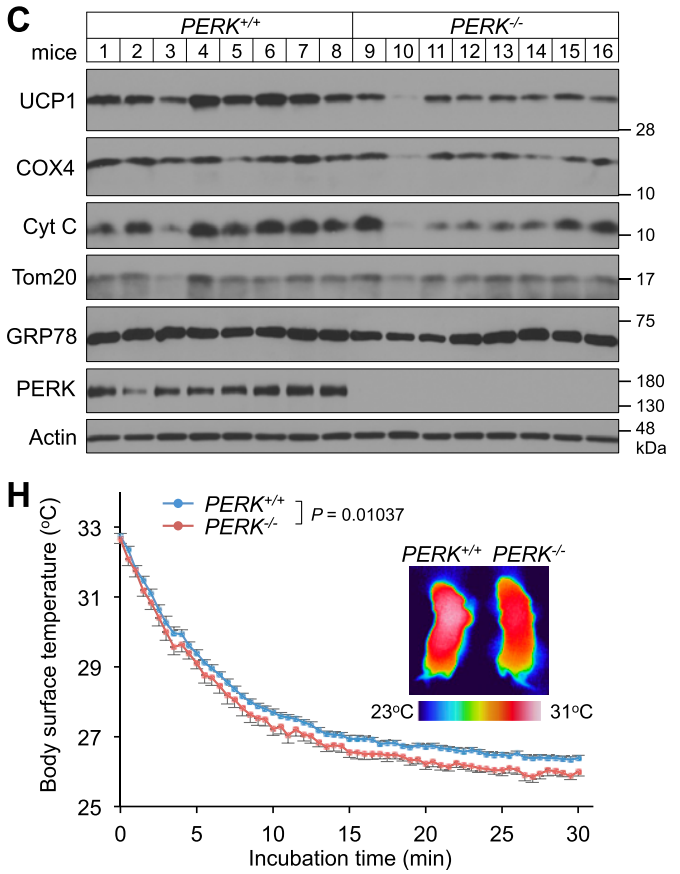

B $\square$ Dense parallel crista morphology $\square$ Sparse crista morphology

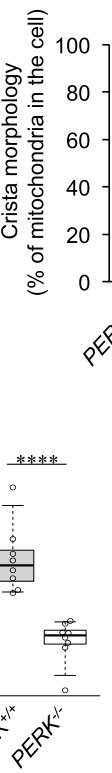

D
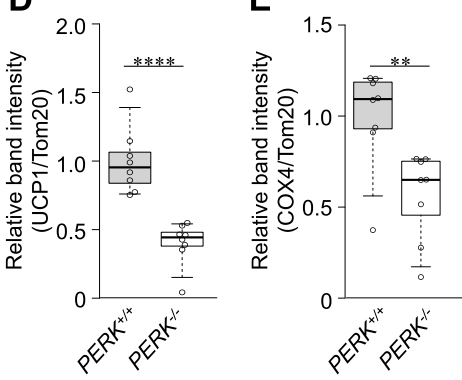

$\mathbf{F}$

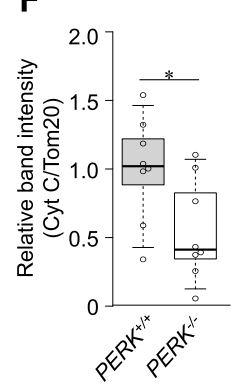

G

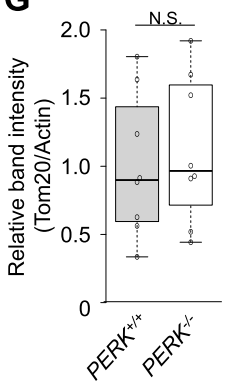

Figure 8. Requirement of PERK for thermogenesis (A) Requirement of PERK for $\beta_{3} A R$ stimulation-induced intracellular thermogenesis. siRNA-transfected brown preadipocytes were infected with retroviruses expressing PERK- $\triangle$ LD or PERK- $\triangle$ LD-3SA, incubated with cellular thermoprobes on day 6 , stimulated with $0.5 \mu \mathrm{M} \mathrm{CL316,243}$ and observed by confocal fluorescence microscopy. The intracellular temperature was analysed by Image] software. Data are shown as the fold change relative to the value at 0 min (sictrl, 20 individual cells; siPERK, 19 individual cells; SIPERK+PERK- $\triangle \mathrm{LD}, 21$ individual cells; and SiPERK+PERK- $\triangle L D-3 S A, 18$ individual cells). (B) Requirement of PERK for dense parallel cristae formation mitochondria in interscapular BAT (iBAT). iBAT was fixed and analysed by electron microscopy. The mitochondria were divided into mitochondria possessing dense parallel cristae and mitochondria possessing sparse cristae and were counted in electron micrographs. The results are shown as described in Fig 2E. Data are shown as the average from $20-30$ (PERK $^{++}$mice) or 22-33 (PERK ${ }^{-1-}$ mice) electron micrographs ( $n=3$ independent experiments). (c) Expression of mitochondrial and ER proteins in iBAT from newborn PERK $K^{++}$or $P E R K^{-/}$mice. Lysates from iBAT were analysed by immunoblotting with the indicated antibodies ( $n=8$ mice). (D, E, F, G) Quantification of uncoupling protein 1 (D), COX4 (E), Cyt C (F), and Tom20 (G) band intensity (see Fig 5 C). Uncoupling protein 1, COX4, and Cyt C were normalized to Tom20. Tom20 was normalized to actin. Data are shown as the fold change relative to the average band intensity of PERK ${ }^{+/+}$mice $(n=8$ independent individuals). (H) Representative thermographic images and dorsal interscapular skin temperature of $P E R K^{+/+}$or $\mathrm{PERK}^{/-}$newborn mice during exposure to $16^{\circ} \mathrm{C}$. The newborn mice, which were incubated at $32^{\circ} \mathrm{C}$, were placed on a $16^{\circ} \mathrm{C}$ plate $(0 \mathrm{~min})$. The back skin temperature was measured by an infrared thermographic camera. $P=$ $0.01037\left(\right.$ PERK $^{+/}, n=13 ;$ PERK $\left.^{/-}, n=8\right) .(\mathbf{A}, \mathbf{D}, \mathbf{E}, \mathbf{F}, \mathbf{G}, \mathbf{H})$ Data information: data are presented as mean \pm SEM. NS; ${ }^{*} P<0.05$; ${ }^{\star \star} P<0.01$; ${ }^{* \star * \star} P<0.0001$ (repeated measures $\operatorname{AnOVA}(A, H), t$ test $(D, E, F, G))$. mitochondrial OXPHOS uncoupler carbonyl cyanide m-chlorophenyl hydrazine (CCCP), endogenous PERK was recognized by PERK-3S $\mathrm{Ab}$ (Fig S6C). Moreover, PERK was required for CCCP-induced thermogenesis in BAs (Fig S6D). Thus, we hypothesize that the secondary events triggered by mitochondrial stress, e.g., generation of reactive oxygen species, influx/efflux of calcium ions, and loss of $\Delta \Psi \mathrm{m}$ may activate the PERK-GABP $\alpha$ axis. However, the fact that the antioxidant reagent $\mathrm{N}$-acetyl cysteine did not inhibit PERK phosphorylation induced by CCCP suggests that reactive oxygen species may not mediate the transduction of mitochondrial stress signals to PERK (data not shown). Perturbation of the cellular lipid composition activated PERK and IRE1 $\alpha$ independently of the UPR (Promlek et al, 2011; Volmer et al, 2013), whereas the phosphorylation status of IRE1 $\alpha$ was not affected on day 2 (Fig $2 A$ and B). We, thus, speculate that PERK phosphorylation might not be triggered by alterations in lipid composition. Identification of the kinase that is activated during BA differentiation and that contributes to PERK phosphorylation may clarify the detailed mechanism by which mitochondrial inner membrane protein biogenesis is regulated in BAs.

Another important question is how PERK regulates mitochondrial inner membrane protein biogenesis and crista formation. Although PERK deficiency had no effect on the areas of ER-mitochondria contact sites (Fig S3H-J), PERK enrichment in the MAM might contribute specifically to parallel crista formation in ER-attached mitochondria (Verfaillie et al, 2012). Because we do not yet have evidence regarding the physiological relevance of the increased areas of ERmitochondria contact sites to PERK-GABP $\alpha$ axis-mediated mitochondrial function, further investigation is necessary. The mRNA expression of Ppargc1a, which encodes the thermogenic coactivator PGC-1 $\alpha$ (Kang et al, 2005), was lower in PERK-deficient BAs than in control BAs (Fig 6A). PGC- $1 \alpha$ binds GABP $\beta 1$ and transcriptionally activates GABP $\alpha$ target genes associated with mitochondrial respiratory complex genes (Wu et al, 1999; Handschin et al, 2007). Because Ppargcia gene expression is regulated by several transcription factors, including ATF2 (Cao et al, 2004), MEF2 (Handschin et al, 2003), and CREB (Herzig et al, 2001), which are known to be targets of GABP $\alpha$ (chromatin immunoprecipitation sequencing datasets, http://amp.pharm.mssm.edu/Harmonizome/) (Rouillard et al, 2016), we reasoned that Ppargc1a may be one of the primary targets of the PERK-GABP $\alpha$ axis. Because the reductions in Cyt $C$, COX4, and UCP1 in PERK-deficient BAs were partially ameliorated by exogenously expressed PGC-1 $\alpha$ (Fig S6E, lane 6), some mitochondrial genes targeted by PERK may be regulated by the cooperation of PGC- $1 \alpha$ with GABP $\alpha$. Because phosphorylation of GABP $\alpha$ and 
GABP $\beta 1$ by ERK and JNK contributes to transcriptional activation of GABP $\alpha$ target genes in skeletal muscle cells (Fromm \& Burden, 2001), PERK or its downstream target kinase may phosphorylate and regulate the GABP $\alpha$ pathway. Another possibility is that abnormal crista formation may occur before reduction in the expression of mitochondrial inner membrane and crista proteins given that there is a correlation between crista formation and mitochondrial inner membrane protein biogenesis. Crista morphology is maintained by cardiolipin synthesis and the formation of crista junctions through the mitochondrial cristae organizing system (MICOS) (Pfanner et al, 2014; Guarani et al, 2015; Anand et al, 2016; Kojima et al, 2019). In BAs and beige adipocytes, cardiolipin synthesis is essential for systemic energy homeostasis mediated by thermogenesis (Sustarsic et al, 2018). Interestingly, our RNA sequencing analysis of HEK293 cells revealed that MIC10, which encodes one of the MICOS components, was reduced by PERK deficiency (Table S2), and analysis of chromatin immunoprecipitation sequencing datasets (Harmonizome) revealed that some MICOS component genes, including MIC10, MIC26, MIC60, and MIC19, are targets of GABP $\alpha$ (Rouillard et al, 2016). One possible explanation is that defective crista junction formation initially triggered by inhibition of MIC10 may attenuate the expression of mitochondrial inner membrane and crista proteins in PERK-deficient BAs, resulting in dysfunction of mitochondria in these BAs.

In conclusion, our findings demonstrate a novel mechanism by which BAs acquire fully developed and functional mitochondria to produce ATP and heat. PERK maintains mitochondrial homeostasis in addition to ER homeostasis through independent mechanisms other than eIF2 $\alpha$-ATF4 axis signalling. Although further investigation is needed to clarify the mechanisms by which PERK is phosphorylated at Ser715, Ser717, and/or Ser719 during BA differentiation, PERK-GABP $\alpha$ axis signalling may be a target through which to increase energy metabolism without affecting ER homeostasis.

\section{Materials and Methods}

\section{Cell culture}

The primary stromal vascular fraction from iBAT of newborn ICR mice was obtained by collagenase digestion. The digested iBAT was filtered through a 100- $\mu \mathrm{m}$ nylon cell strainer, and the cells were isolated by centrifugation (156g) for $5 \mathrm{~min}$. The cell pellet was washed with PBS and cultured with DMEM supplemented with $20 \%$ FBS. The cells were seeded, grown to confluence (designated day 2) and cultured with differentiation induction medium (DMEM [SigmaAldrich] supplemented with 20\% FBS, 20 nM insulin [Sigma-Aldrich], $1 \mathrm{nM}$ triiodo-L-thyronine [T3; Sigma-Aldrich], $5 \mu \mathrm{M}$ dexamethasone, $0.125 \mathrm{mM}$ indomethacin [Sigma-Aldrich], $0.5 \mathrm{mM}$ IBMX [SigmaAldrich], and $1 \mu \mathrm{M}$ rosiglitazone [Sigma-Aldrich]) on day 0 . After differentiation induction, the cells were cultured with differentiation enhancement medium (DMEM supplemented with 20\% FBS, 20 nM insulin, and 1 nM T3) on days 2 and 4 . HEK293 cells were maintained in DMEM containing 10\% FBS and penicillin-streptomycin (glycolysis conditions). For habituation to OXPHOS conditions, HEK293 cells were cultured with glucose-free DMEM (Nacalai tesque) containing 10\% FBS, $1 \mathrm{mM}$ sodium pyruvate, $10 \mathrm{mM}$ galactose and penicillin-streptomycin and incubated for 12 or $24 \mathrm{~h}$.

\section{Mice}

ICR mice (SLC) were raised under specific pathogen-free conditions at the animal facility of University of Miyazaki. The $P E R K^{-/-}$mice have been previously described (Zhang et al, 2002). Newborn PERK ${ }^{-1}$ mice were obtained after mating $P E R K^{+-}$male and female mice. The $\mathrm{PERK}^{+/-}$mice were maintained under specific pathogen-free conditions at the Institute of Genome Research of Tokushima University. All animal experiments were approved by the University of Miyazaki and Tokushima University and were performed in accordance with the appropriate institutional guidelines.

\section{Plasmid and siRNA transfection}

AARE-luc plasmid has been previously described (Miyake et al, 2016). A $\mathrm{GABP} \alpha$-binding element luciferase promoter reporter construct (GABP $\alpha$-luc) was provided by Dr Nono Tomita (University of Tokyo, Tokyo, Japan) (Hayashi et al, 2007). pRL-CMV was purchased from Promega. The cells were transfected using FuGENE6 (Roche) or polyethylenimine (PEI)-Max (Polysciences). siRNA transfection was performed using Lipofectamine RNAiMAX reagent (Invitrogen). siRNAs (mouse PERK, MSS203819, MSS203821, and NM_010121.2_stealth_3673; mouse GABP $\alpha$, \#1-MSS274443, and \#2-MSS274444; mouse IRE1 $\alpha$, \#1MSS234443, and \#2-MSS234445; and mouse ATF6, \#1-MSS213140, and \#2-MSS279117) were purchased from Invitrogen. Negative Control Med GC Duplex was used as the control. The PERK siRNA (siPERK) MSS203821 was used in Figs 3A-F, 4A-E, 6A-C, 7A, S3A-J, S5E and F, and S6E. The siPERK MSS2023819 was used in Figs 5B and C, 5E-G, $7 C$ and D, 8A, S4A and B, and S6D. NM_010121.2_stealth_3673 was used in Fig $5 \mathrm{H}$. siRNAs are listed in Table $\mathrm{S3}$.

\section{Generation of retrovirus and infection}

PERK-ALD-Flag, PERK- $L$ LD-KA-Flag, PERK- $\triangle$ LD-3SA-Flag, PERK-Flag, PERK-KA-Flag, PERK-3SA-Flag, Flag-GABP $\alpha$, Flag-GABP $\beta 1$, and PGC$1 \alpha$-Flag were constructed into the pMXs-IP plasmid. Recombinant retroviruses were produced by transient transfection of retroviral packaging cells (Platinum-GP) using PEI-Max, and primary brown preadipocytes were infected with recombinant retroviruses in DMEM containing $7.5 \mu \mathrm{g} / \mathrm{ml}$ polybrene (Sigma-Aldrich) for $24 \mathrm{~h}$.

\section{Generation of CRISPR KO and knock-in cells}

PERK-KO or PERK-Flag-knock-in HEK293 cells were generated using the CRISPR/CRISPR-associated 9 (Cas9) system. gRNAs for KO (5'GATCCTGTTCTTCTTTTACAC-3' or 5'-GACTGCAATTATGCTATCAAG-3') and knock-in (5'-AGCAATTAGCCTTAAGTTGT-3' or 5'-GTTGTGCTAGCAACCCTAAT-3') were ligated into pX330 or pX335 vectors (Addgene). To generate knock-in cells, $5^{\prime}$ arm and $3^{\prime}$ arm sequences were amplified from genomic DNA by PCR using $5^{\prime}$ arm primers (5'-TTCAGCCTAATGTCCAGTGT-3' and 5'-ATTGCTTGGCAAAGGGCTA-3') and 3' arm primers (5'-CTTCTTAGAATATGCCTGTC- $3^{\prime}$ and 5'-ATGACTCTTCCTAGATCTA-3'). These arm sequences were inserted together with $3 \times$ Flag, Poly $A, \beta$ actin promoter, and Neo sequences in the empty 
vectors, which were used as donor vectors. The plasmids were transfected into HEK293 cells using PEI-Max, and single cells were seeded in a 96-well plate or selected by G418. These clones were analysed by IB.

\section{Intracellular ATP assay}

An ATP assay was performed using an ATP Bioluminescence Assay Kit CLS II (Roche). The intensity of luminescence was measured with a TD-20/20 luminometer (Promega). The ATP content was normalized to the cell number. The amount of oligomycin A (Sigma-Aldrich)insensitive intracellular ATP was used to calculate glycolysisdependent ATP production. OXPHOS-dependent ATP production was calculated from the amounts of total intracellular ATP and glycolysis-produced ATP (Ojaimi et al, 2002; Yang et al, 2014).

\section{Measurement of cellular basal OCR and ECAR}

Primary brown preadipocytes were seeded in an XFp cell culture plate at 20,000 cells/well and then differentiated. On day 0, 2, 4, and 6 , the culture medium was replaced with an XF Assay Medium supplemented $10 \mathrm{mM}$ glucose, $1 \mathrm{mM}$ pyruvate, and $2 \mathrm{mM}$ glutamine. After 60 min incubation at $37^{\circ} \mathrm{C}$ in $\mathrm{CO}_{2}$-free incubator, the OCR and ECAR were measured using an XFp extracellular flux analyser (Agilent Technologies) and XF Cell Mito stress test kit, and then the basal OCR and energy phenotype profiling (basal OCR/ECAR) were calculated. The mitochondrial stress test was performed according to the manufacturer's instructions ( $8 \mu \mathrm{M}$ oligomycin $\mathrm{A}, 1 \mu \mathrm{M}$ carbonyl cyanide-p-trifluoromethoxyphenylhydrazone, $1 \mu \mathrm{M}$ rotenone, and $1 \mu \mathrm{M}$ antimycin $\mathrm{A}$ ). The data were normalized by total protein content.

\section{Luciferase assay}

siRNA-transfected brown preadipocytes were co-transfected with $10 \mu \mathrm{g}$ of AARE-luc or GABP $\alpha$-luc and $1 \mu \mathrm{g}$ of Renilla-luc plasmids and infected with retroviruses expressing Venus, PERK-Flag, PERKKA-Flag, PERK-3SA-Flag, PERK- $L$ LD-Flag, PERK- $L$ LD-KA-Flag, or PERK- $\triangle$ LD-3SA-Flag. A luciferase assay was performed using a DualLuciferase Reporter Assay System (Promega). The promoter activity was normalized using Renilla reporter values for transfection efficiency according to the manufacturer's protocol.

\section{IB and immunoprecipitation}

IB and immunoprecipitation experiments have been described in detail (Kadowaki et al, 2015). Cells and iBAT were lysed in lysis buffer (20 mM Tris- $\mathrm{HCl}, \mathrm{pH}$ 7.5, 150 mM NaCl, 5 mM EGTA, 1\% Triton X-100, $\beta$-glycerophosphate, and $1 \%$ sodium deoxycholate) containing $1 \mathrm{mM}$ phenylmethylsulfonyl fluoride, $1 \mathrm{mM} \mathrm{Na}_{3} \mathrm{VO}_{4}$, and $50 \mathrm{mM} \mathrm{NaF}$ on ice. The proteins were separated by SDS-PAGE, blotted onto PVDF membranes and blocked in TBS/T containing 5\% dry milk. The membranes were incubated with antibodies and detected by an ECL system. The immunoprecipitation experiments were performed using an anti-Flag M2 antibody affinity gel (Sigma-Aldrich). After washing the gels, the immunoprecipitates were detected by IB. Band intensity was measured by ImageQuant TL (GE Healthcare) or
Imagej software (National Institutes of Health). Antibodies are listed in Table S3.

\section{$\lambda$ phosphatase treatment}

Cell lysates were treated with 2 units of $\lambda$ phosphatase (New England BioLabs) at $30^{\circ} \mathrm{C}$ for $30 \mathrm{~min}$. SDS sample buffer was added, and the samples were incubated at $98^{\circ} \mathrm{C}$ for $5 \mathrm{~min}$.

\section{Puromycin labeling assay}

Cells were treated with $10 \mu \mathrm{g} / \mathrm{ml}$ puromycin for $10 \mathrm{~min}$, immediately washed with PBS and lysed. The puromycin-labeled proteins were analysed by IB with an anti-puromycin antibody. Antibodies are listed in Table S3.

\section{Quantitative PCR analysis}

Total RNA was isolated from cells using RNAiso (Takara). RT was performed using RevaTra Ace qPCR RT Master Mix with gDNA Remover (TOYOBO). qPCR was performed with a StepOnePlus RealTime PCR System (Applied Biosystems) with SYBR Green PCR Master Mix (Kapa Biosystems). The expression level of S18 mRNA was used as a normalization control. The primer sequences are shown in Table S3.

\section{Mitochondrial DNA quantification}

Cells were digested with proteinase $\mathrm{K}$ for $2 \mathrm{~h}$ at $55^{\circ} \mathrm{C}$, and total DNA was extracted with phenol/chloroform. Quantification of genomic DNA ( $\beta$-globin) and mtDNA (COX2) was performed using qPCR. The primer sequences are provided in Table S3.

\section{RNA sequencing}

Control and PERK-deficient differentiating cells were cultured for 16 or $24 \mathrm{~h}$ in differentiating enhancement medium on day 2. WT and PERK-deficient HEK293 cells were incubated with OXPHOS medium for $12 \mathrm{~h}$. Total RNA was isolated using an RNeasy kit (Invitrogen), and the quality (RNA integrity number: 10) was measured using a Bioanalyser 2100 (Agilent Technologies). Purification of mRNA was performed using NEBNext poly (A) magnetic beads (New England BioLabs). DNA libraries were prepared with an NEBNext DNA Library Prep Master Mix Set for Illumina and NEBNext Singleplex Oligos for Illumina (New England BioLabs) according to the manufacturer's instructions. The sequence reads from the RNA sequencing analysis were mapped to the human reference genome (GRCm38/mm10 or GRCh37/hg19) using TopHat version 2. The mapped sequences were converted to expression levels (fragments per kilobase of exon per million reads mapped, FPKM) using Cufflinks and Cuffdiff. The results are expressed as the log2 fold change relative to the levels in control differentiating cells or WT HEK293 cells. Gene Ontology analysis was performed using PANTHER (http://geneontology.org). A binding motif assay was performed by MEME motif analysis (http://meme.sdsc.edu) (Bailey et al, 2009). 


\section{LC-MS/MS-based phosphoproteomic analysis and sample preparation}

To identify the phosphorylation sites of PERK in differentiating cells, siPERK-transfected primary brown preadipocytes were infected with retroviruses expressing Flag-tagged PERK- $\triangle$ LD-KA, and extraction was performed after $12 \mathrm{~h}$ of culture with differentiation enhancement medium on day 2. PERK- $\triangle$ LD-KA was purified from the cell lysates using M2-Flag beads and eluted with $3 \times$ Flag peptides. The Purified PERK- $\triangle$ LD-KA was digested with Glu-C (Promega) at $37^{\circ} \mathrm{C}$ for $18 \mathrm{~h}$. The peptides were desalted with a Monospin C18 column (GL Sciences), and then the phosphopeptides were enriched using a High-Select Fe-NTA Phosphopeptide Enrichment Kit (Thermo Fisher Scientific). The phosphopeptides were analysed by nanoscale LC-MS/MS using an Ultimate300 RSLCnano (Thermo Fisher Scientific) and a Q-Exactive mass spectrometer (Thermo Fisher Scientific). The LC separation was performed using an EASYSpray column $(75-\mu \mathrm{m}$ inner diameter, $25 \mathrm{~cm}$ packed with $\mathrm{C} 18$ reversed-phase resin). The MS/MS was performed in a datadependent fashion with a top 10 method. For peptide mapping and identification of phosphorylation sites, raw data were analysed using Proteome Discoverer and searched for peptides containing phosphoserine, phosphothreonine, or phosphotyrosine against the MASCOT and SEQUEST HT search engine.

\section{Immunofluorescence staining}

Endogenous ER proteins and mitochondrial proteins were detected by anti-KDEL and anti-Tom20 antibodies. On sterile glass coverslips, primary brown preadipocytes were cultured and differentiation was induced, and the cells were harvested on day 6 . After fixing in $4 \%$ paraformaldehyde at room temperature for $25 \mathrm{~min}$, the cells were washed with PBS, permeabilized in $0.2 \%$ Triton X-100, blocked with $1 \% \mathrm{BSA}$ in PBS for $1 \mathrm{~h}$, and then incubated with primary antibodies for $12 \mathrm{~h}$ at $4^{\circ} \mathrm{C}$. After washing with PBS, the cells were incubated with Alexa Fluor 488- or Alexa Fluor 546-conjugated secondary antibodies (Thermo Fisher Scientific) for $1.5 \mathrm{~h}$ at room temperature. The cells were washed with PBS and mounted in VECTASHIELD mounting medium containing DAPI (Vector Laboratories). Immunofluorescence images were obtained using fluorescence microscopy and confocal laser microscopy (TSC-SP8; Leica). Quantitative analysis of the cell images was performed using ImageJ software. Antibodies are listed in Table S3.

\section{Electron microscopy}

Cells and iBAT were washed with PBS and fixed in modified Karnovsky's fixative (3\% glutaraldehyde and $1.6 \%$ paraformaldehyde in $0.1 \%$ sodium cacodylate, $\mathrm{pH} 7.4$ ) before being fixed in $1 \%$ osmium tetroxide. After dehydration with an ethanol series (50\%, 70\%, 80\%, $90 \%, 95 \%$, and $100 \%$ ), ultrathin sections (70-90 nm) were cut, stained, with uranyl acetate and imaged using an HT7700 transmission electron microscope (HITACHI) at $100 \mathrm{kV}$. ER-mitochondria contact sites were defined as sites with a distance of $<30 \mathrm{~nm}$ between the membranes of the two organelles. Quantification of the ER-mitochondria distance and contact length was performed using ImageJ software.

\section{Oil red 0 staining}

Primary BAs were fixed with $10 \%$ formalin in PBS for $10 \mathrm{~min}$, washed with PBS, rinsed with $60 \%$ isopropanol, and stained with oil red $O$ solution. After washing with $60 \%$ isopropanol and PBS, images were obtained. To quantify the amounts of LDs, oil red 0 (Sigma-Aldrich)-stained cells were lysed in 100\% isopropanol containing Nonidet P-40 for 10 min. Absorbance of isolated oil red O was determined by a spectrophotometer (492 $\mathrm{nm}$ wavelength).

\section{$\Delta \Psi$ m assay}

For the $\Delta \Psi \mathrm{m}$ assay, the cells were incubated with MITO-ID Membrane Potential Detection Reagent and Necrosis Detection Reagent (Enzo Life Sciences) for $15 \mathrm{~min}$ at room temperature and analysed by confocal fluorescence microscopy or flow cytometry (BD FACSVerse; BD Biosciences).

\section{Intracellular thermogenesis assay}

An intracellular thermogenesis assay was performed using two different thermoprobes. BAs (day 6) were washed with PBS and 5\% glucose solution and then incubated with $0.04 \mathrm{wt} / \mathrm{vol} \%$ cellular thermoprobe (FDV-0004; Funakoshi) for $10 \mathrm{~min}$. The fluorescence intensity of single cells was observed before and after stimulation with $0.5 \mu \mathrm{M}$ CL316,243 (Sigma-Aldrich) by confocal laser microscopy (TSC-SP8; Leica). A hydrophilic fluorescent nanogel thermometer (Gota et al, 2009) was microinjected into the cytosol of BAs. The fluorescence intensity of single cells was observed before and after stimulation with $10 \mu \mathrm{M}$ CCCP (Sigma-Aldrich) under an IX70 inverted microscope (Olympus) equipped with an objective lens (60x, UplanApo N.A. 1.40; Olympus). A cooled charge-coupled device camera (ORCA-ER) was used to acquire cell images.

\section{Thermographic analysis}

Newborn mice were incubated at $32^{\circ} \mathrm{C}$ and placed on a $16^{\circ} \mathrm{C}$ plate, and thermographic images were obtained with an infrared thermographic camera (TVS-200; Nippon Avionics). Thermographic analysis was performed using PE Professional (GORATEC).

\section{Histopathological analysis}

Tissue sections of 4- $\mu \mathrm{m}$ thickness were stained with haematoxylin and eosin, and images were obtained by microscopy (DM1000 LED; Leica).

\section{Quantification and statistical analysis}

The data are presented as the mean \pm standard error, and statistical analysis was performed using $t$ test and repeated measures ANOVA to compare data in different groups. Statistical analysis was performed by using EZR software version1.30 (Kanda, 2013). 


\section{Data and software availability}

RNA-seq data used in this study have been deposited in the NCBI Gene Expression Omnibus under accession number GSE113572 and GSE132598. IB experiments were repeated at least three times independently, and the representative data are shown. All datasets are available from the corresponding author upon reasonable request.

\section{Supplementary Information}

Supplementary Information is available at https://doi.org/10.26508/lsa. 201900576.

\section{Acknowledgements}

We thank N Tomita (University of Tokyo) and K Mikoshiba (RIKEN) for providing us with plasmids. We also thank $\mathrm{K}$ Imaizumi (Hiroshima University), M Nakamura Tokyo Medical and Dental University (TMDU), N Ishihara (Osaka University), A Sawaguchi, Y Goto, A Nakatake, and laboratory members (University of Miyazaki) for technical assistance and discussion. This study was supported by AMED (Japan Agency for Medical Research and Development) (grant number JP19gm5010001) (H Ichijo), Ministry of Education, Culture, Sports, Science and Technology (MEXT) KAKENHI (grant number JP18H03995 [H Ichijo], 18K06916 [H Kato], 17H06419 [H Nishitoh], 18H02973 [H Nishitoh], and 18H04699 [H Nishitoh]) from the Japan Society for the Promotion of Science, Mitsubishi Foundation, Uehara Memorial Foundation, Astellas Foundation for Research on Metabolic Disorders, Takeda Science Foundation, Project for Creation of Research Platforms and Sharing of Advanced Research Infrastructure, and Nanken-Kyoten, TMDU (to $\mathrm{H}$ Nishitoh).

\section{Author Contributions}

H Kato: conceptualization, funding acquisition, investigation, and writing-original draft.

K Okabe: resources and investigation.

M Miyake: resources.

K Hattori: methodology.

T Fukaya: resources and investigation.

$K$ Tanimoto: resources and investigation.

$S$ Beini: investigation.

M Mizuguchi: investigation and methodology.

S Torii: methodology.

S Arakawa: methodology.

M Ono: investigation and methodology.

Y Saito: methodology.

T Sugiyama: software and formal analysis.

T Funatsu: supervision and methodology.

K Sato: supervision and methodology.

S Shimizu: supervision and methodology.

S Oyadomari: resources.

$\mathrm{H}$ Ichijo: supervision and methodology.

$\mathrm{H}$ Kadowaki: conceptualization, supervision, and writing-review and editing.
H Nishitoh: conceptualization, data curation, supervision, funding acquisition, project administration, and writing-original draft, review, and editing.

\section{Conflict of Interest Statement}

The authors declare that they have no conflict of interest.

\section{References}

Anand R, Strecker V, Urbach J, Wittig I, Reichert AS (2016) Mic13 is essential for formation of crista junctions in mammalian cells. PLoS One 11: e0160258. doi:10.1371/journal.pone.0160258

Bailey TL, Boden M, Buske FA, Frith M, Grant CE, Clementi L, Ren J, Li WW, Noble WS (2009) MEME SUITE: Tools for motif discovery and searching. Nucleic Acids Res 37: W202-W208. doi:10.1093/nar/gkp335

Balsa E, Soustek MS, Thomas A, Cogliati S, Garcia-Poyatos C, Martin-Garcia E, Jedrychowski M, Gygi SP, Enriquez JA, Puigserver P (2019) ER and nutrient stress promote assembly of respiratory chain supercomplexes through the PERK-elF2alpha axis. Mol Cell 74: 877-890. doi:10.1016/j.molcel.2019.03.031

Bartelt A, Widenmaier SB, Schlein C, Johann K, Goncalves RLS, Eguchi K, Fischer AW, Parlakgul G, Snyder NA, Nguyen TB, et al (2018) Brown adipose tissue thermogenic adaptation requires Nrf1-mediated proteasomal activity. Nat Med 24: 292-303. doi:10.1038/nm.4481

Benador IY, Veliova M, Mahdaviani K, Petcherski A, Wikstrom JD, Assali EA, Acin-Perez R, Shum M, Oliveira MF, Cinti S, et al (2018) Mitochondria bound to lipid droplets have unique bioenergetics, composition, and dynamics that support lipid droplet expansion. Cell Metab 27: 869-885.e6. doi:10.1016/j.cmet.2018.03.003

Bertolotti A, Zhang Y, Hendershot LM, Harding HP, Ron D (2000) Dynamic interaction of BiP and ER stress transducers in the unfolded-protein response. Nat Cell Biol 2: 326-332. doi:10.1038/35014014

Bouman L, Schlierf A, Lutz AK, Shan J, Deinlein A, Kast J, Galehdar Z, Palmisano V, Patenge N, Berg D, et al (2011) Parkin is transcriptionally regulated by ATF4: Evidence for an interconnection between mitochondrial stress and ER stress. Cell Death Differ 18: 769-782. doi:10.1038/ cdd.2010.142

Cannon B, Nedergaard J (2004) Brown adipose tissue: Function and physiological significance. Physiol Rev 84: 277-359. doi:10.1152/ physrev.00015.2003

Cao W, Daniel KW, Robidoux J, Puigserver P, Medvedev AV, Bai X, Floering LM, Spiegelman BM, Collins S (2004) p38 mitogen-activated protein kinase is the central regulator of cyclic AMP-dependent transcription of the brown fat uncoupling protein 1 gene. Mol Cell Biol 24: 3057-3067. doi:10.1128/mcb.24.7.3057-3067.2004

Chen JQ, Cammarata PR, Baines CP, Yager JD (2009) Regulation of mitochondrial respiratory chain biogenesis by estrogens/estrogen receptors and physiological, pathological and pharmacological implications. Biochim Biophys Acta 1793: 1540-1570. doi:10.1016/ j.bbamcr.2009.06.001

Chen Y, Ikeda K, Yoneshiro T, Scaramozza A, Tajima K, Wang Q, Kim K, Shinoda K, Sponton CH, Brown Z, et al (2019) Thermal stress induces glycolytic beige fat formation via a myogenic state. Nature 565: 180-185. doi:10.1038/s41586-018-0801-z

de Meis L, Ketzer LA, da Costa RM, de Andrade IR, Benchimol M (2010) Fusion of the endoplasmic reticulum and mitochondrial outer membrane in rats brown adipose tissue: Activation of thermogenesis by Ca2+. PLOS One 5: e9439. doi:10.1371/journal.pone.0009439 
Dorn GW 2nd, Vega RB, Kelly DP (2015) Mitochondrial biogenesis and dynamics in the developing and diseased heart. Genes Dev 29: 1981-1991. doi:10.1101/gad.269894.115

Feldmann HM, Golozoubova V, Cannon B, Nedergaard J (2009) UCP1 ablation induces obesity and abolishes diet-induced thermogenesis in mice exempt from thermal stress by living at thermoneutrality. Cell Metab 9: 203-209. doi:10.1016/j.cmet.2008.12.014

Friedman JR, Lackner LL, West M, DiBenedetto JR, Nunnari J, Voeltz GK (2011) ER tubules mark sites of mitochondrial division. Science 334: 358-362. doi:10.1126/science. 1207385

Fromm L, Burden SJ (2001) Neuregulin-1-stimulated phosphorylation of GABP in skeletal muscle cells. Biochemistry 40: 5306-5312. doi:10.1021/ bi002649m

Golic I, Velickovic K, Markelic M, Stancic A, Jankovic A, Vucetic M, Otasevic V, Buzadzic B, Korac B, Korac A (2014) Calcium-induced alteration of mitochondrial morphology and mitochondrial-endoplasmic reticulum contacts in rat brown adipocytes. Eur J Histochem 58: 2377. doi:10.4081/ejh.2014.2377

Gota C, Okabe K, Funatsu T, Harada Y, Uchiyama S (2009) Hydrophilic fluorescent nanogel thermometer for intracellular thermometry. I Am Chem Soc 131: 2766-2767. doi:10.1021/ja807714j

Guarani V, McNeill EM, Paulo JA, Huttlin EL, Frohlich F, Gygi SP, Van Vactor D, Harper JW (2015) QIL1 is a novel mitochondrial protein required for MICOS complex stability and cristae morphology. Elife 4: e06265. doi:10.7554/eLife.06265

Hamasaki M, Furuta N, Matsuda A, Nezu A, Yamamoto A, Fujita N, Oomori H, Noda T, Haraguchi T, Hiraoka Y, et al (2013) Autophagosomes form at ER-mitochondria contact sites. Nature 495: 389-393. doi:10.1038/ nature11910

Handschin C, Kobayashi YM, Chin S, Seale P, Campbell KP, Spiegelman BM (2007) PGC-1alpha regulates the neuromuscular junction program and ameliorates Duchenne muscular dystrophy. Genes Dev 21: 770-783. doi:10.1101/gad.1525107

Handschin C, Rhee J, Lin J, Tarr PT, Spiegelman BM (2003) An autoregulatory loop controls peroxisome proliferator-activated receptor gamma coactivator 1alpha expression in muscle. Proc Natl Acad Sci U S A 100: 7111-7116. doi:10.1073/pnas.1232352100

Harding HP, Novoa I, Zhang Y, Zeng H, Wek R, Schapira M, Ron D (2000) Regulated translation initiation controls stress-induced gene expression in mammalian cells. Mol Cell 6: 1099-1108. doi:10.1016/ s1097-2765(00)00108-8

Harding HP, Zhang Y, Ron D (1999) Protein translation and folding are coupled by an endoplasmic-reticulum-resident kinase. Nature 397: 271-274. doi:10.1038/16729

Harms M, Seale P (2013) Brown and beige fat: Development, function and therapeutic potential. Nat Med 19: 1252-1263. doi:10.1038/nm.3361

Hayashi R, Ueda T, Farwell MA, Takeuchi N (2007) Nuclear respiratory factor 2 activates transcription of human mitochondrial translation initiation factor 2 gene. Mitochondrion 7: 195-203. doi:10.1016/j.mito.2006.10.005

Herzig S, Long F, Jhala US, Hedrick S, Quinn R, Bauer A, Rudolph D, Schutz G, Yoon C, Puigserver $\mathrm{P}$, et al (2001) CREB regulates hepatic gluconeogenesis through the coactivator PGC-1. Nature 413: 179-183. doi:10.1038/35093131

Hirabayashi Y, Kwon SK, Paek H, Pernice WM, Paul MA, Lee J, Erfani P, Raczkowski A, Petrey DS, Pon LA, et al (2017) ER-mitochondria tethering by PDZD8 regulates $\mathrm{Ca}(2+)$ dynamics in mammalian neurons. Science 358: 623-630. doi:10.1126/science.aan6009

Hodges MR, Tattersall GJ, Harris MB, McEvoy SD, Richerson DN, Deneris ES, Johnson RL, Chen ZF, Richerson GB (2008) Defects in breathing and thermoregulation in mice with near-complete absence of central serotonin neurons. J Neurosci 28: 2495-2505. doi:10.1523/ JNEUROSCI.4729-07.2008
Hori O, Ichinoda F, Tamatani T, Yamaguchi A, Sato N, Ozawa K, Kitao Y, Miyazaki M, Harding HP, Ron D, et al (2002) Transmission of cell stress from endoplasmic reticulum to mitochondria: Enhanced expression of Lon protease. J Cell Biol 157: 1151-1160. doi:10.1083/jcb.200108103

Kadowaki H, Nagai A, Maruyama T, Takami Y, Satrimafitrah P, Kato H, Honda A, Hatta T, Natsume T, Sato T, et al (2015) Pre-emptive quality control protects the ER from protein overload via the proximity of ERAD components and SRP. Cell Rep 13: 944-956. doi:10.1016/ j.celrep.2015.09.047

Kajimura S, Saito M (2014) A new era in brown adipose tissue biology: Molecular control of brown fat development and energy homeostasis. Annu Rev Physiol 76: 225-249. doi:10.1146/annurev-physiol-021113170252

Kanda Y (2013) Investigation of the freely available easy-to-use software "EZR" for medical statistics. Bone Marrow Transplant 48: 452-458. doi:10.1038/bmt.2012.244

Kang S, Bajnok L, Longo KA, Petersen RK, Hansen JB, Kristiansen K, MacDougald OA (2005) Effects of Wnt signaling on brown adipocyte differentiation and metabolism mediated by PGC-1alpha. Mol Cell Biol 25: 1272-1282. doi:10.1128/MCB.25.4.1272-1282.2005

Kato H, Nishitoh H (2015) Stress responses from the endoplasmic reticulum in cancer. Front Oncol 5: 93. doi:10.3389/fonc.2015.00093

Kojima R, Kakimoto Y, Furuta S, Itoh K, Sesaki H, Endo T, Tamura Y (2019) Maintenance of cardiolipin and crista structure requires cooperative functions of mitochondrial dynamics and phospholipid transport. Cell Rep 26: 518-528.e6. doi:10.1016/j.celrep.2018.12.070

Kornmann B, Currie E, Collins SR, Schuldiner M, Nunnari J, Weissman JS, Walter P (2009) An ER-mitochondria tethering complex revealed by a synthetic biology screen. Science 325: 477-481. doi:10.1126/ science. 1175088

Lebeau J, Saunders JM, Moraes VWR, Madhavan A, Madrazo N, Anthony MC, Wiseman RL (2018) The PERK arm of the unfolded protein response regulates mitochondrial morphology during acute endoplasmic reticulum stress. Cell Rep 22: 2827-2836. doi:10.1016/ j.celrep.2018.02.055

Lever JD (1957) The fine structure of brown adipose tissue in the rat with observations on the cytological changes following starvation and adrenalectomy. Anat Rec 128: 361-377. doi:10.1002/ar.1091280302

Liu X, Rossmeisl M, McClaine J, Riachi M, Harper ME, Kozak LP (2003) Paradoxical resistance to diet-induced obesity in UCP1-deficient mice. J Clin Invest 111: 399-407. doi:10.1172/JCl1573710.1172/jci200315737

Matthias A, Ohlson KB, Fredriksson JM, Jacobsson A, Nedergaard J, Cannon B (2000) Thermogenic responses in brown fat cells are fully UCP1dependent. UCP2 or UCP3 do not substitute for UCP1 in adrenergically or fatty scid-induced thermogenesis. J Biol Chem 275: 25073-25081. doi:10.1074/jbc.M000547200

Miyake M, Nomura A, Ogura A, Takehana K, Kitahara Y, Takahara K, Tsugawa K, Miyamoto C, Miura N, Sato R, et al (2016) Skeletal muscle-specific eukaryotic translation initiation factor 2alpha phosphorylation controls amino acid metabolism and fibroblast growth factor 21mediated non-cell-autonomous energy metabolism. FASEB J 30: 798-812. doi:10.1096/fj.15-275990

Napolitano L, Fawcett D (1958) The fine structure of brown adipose tissue in the newborn mouse and rat. J Biophys Biochem Cytol 4: 685-692. doi:10.1083/jcb.4.6.685

Ojaimi J, Pan J, Santra S, Snell WJ, Schon EA (2002) An algal nucleus-encoded subunit of mitochondrial ATP synthase rescues a defect in the analogous human mitochondrial-encoded subunit. Mol Biol Cell 13: 3836-3844. doi:10.1091/mbc.E02-05-0306

Pfanner N, van der Laan M, Amati P, Capaldi RA, Caudy AA, Chacinska A, Darshi M, Deckers M, Hoppins S, Icho T, et al (2014) Uniform nomenclature for the mitochondrial contact site and cristae organizing system. J Cell Biol 204: 1083-1086. doi:10.1083/jcb.201401006 
Promlek T, Ishiwata-Kimata Y, Shido M, Sakuramoto M, Kohno K, Kimata Y (2011) Membrane aberrancy and unfolded proteins activate the endoplasmic reticulum stress sensor Ire1 in different ways. $\mathrm{Mol}$ Biol Cell 22: 3520-3532. doi:10.1091/mbc.E11-04-0295

Rainbolt TK, Atanassova N, Genereux JC, Wiseman RL (2013) Stress-regulated translational attenuation adapts mitochondrial protein import through Tim17A degradation. Cell Metab 18: 908-919. doi:10.1016/ j.cmet.2013.11.006

Rizzuto R, Pinton P, Carrington W, Fay FS, Fogarty KE, Lifshitz LM, Tuft RA, Pozzan T (1998) Close contacts with the endoplasmic reticulum as determinants of mitochondrial Ca2+ responses. Science 280: 1763-1766. doi:10.1126/science.280.5370.1763

Rouillard AD, Gundersen GW, Fernandez NF, Wang Z, Monteiro CD, McDermott MG, Ma'ayan A (2016) The harmonizome: A collection of processed datasets gathered to serve and mine knowledge about genes and proteins. Database 2016: 1-16. doi:10.1093/database/baw100

Sustarsic EG, Ma T, Lynes MD, Larsen M, Karavaeva I, Havelund JF, Nielsen CH, Jedrychowski MP, Moreno-Torres M, Lundh M, et al (2018) Cardiolipin synthesis in brown and beige fat mitochondria is essential for systemic energy homeostasis. Cell Metab 28: 159-174.e11. doi:10.1016/ j.cmet.2018.05.003

Susulic VS, Frederich RC, Lawitts J, Tozzo E, Kahn BB, Harper ME, HimmsHagen J, Flier JS, Lowell BB (1995) Targeted disruption of the beta 3adrenergic receptor gene. J Biol Chem 270: 29483-29492. doi:10.1074/ jbc.270.49.29483

Vattem KM, Wek RC (2004) Reinitiation involving upstream ORFs regulates ATF4 mRNA translation in mammalian cells. Proc Natl Acad Sci U S A 101: 11269-11274. doi:10.1073/pnas.0400541101

Verfaillie T, Rubio N, Garg AD, Bultynck G, Rizzuto R, Decuypere JP, Piette J, Linehan C, Gupta S, Samali A, et al (2012) PERK is required at the ER- mitochondrial contact sites to convey apoptosis after ROS-based ER stress. Cell Death Differ 19: 1880-1891. doi:10.1038/cdd.2012.74

Volmer R, van der Ploeg K, Ron D (2013) Membrane lipid saturation activates endoplasmic reticulum unfolded protein response transducers through their transmembrane domains. Proc Natl Acad Sci U S A 110: 4628-4633. doi:10.1073/pnas.1217611110

Wu Z, Puigserver P, Andersson U, Zhang C, Adelmant G, Mootha V, Troy A, Cinti S, Lowell B, Scarpulla RC, et al (1999) Mechanisms controlling mitochondrial biogenesis and respiration through the thermogenic coactivator PGC-1. Cell 98: 115-124. doi:10.1016/S0092-8674(00)80611-X

Yang ZF, Drumea K, Mott S, Wang J, Rosmarin AG (2014) GABP transcription factor (nuclear respiratory factor 2 ) is required for mitochondrial biogenesis. Mol Cell Biol 34: 3194-3201. doi:10.1128/MCB.00492-12

Zeng X, Ye M, Resch JM, Jedrychowski MP, Hu B, Lowell BB, Ginty DD, Spiegelman BM (2019) Innervation of thermogenic adipose tissue via a calsyntenin 3beta-S100b axis. Nature 569: 229-235. doi:10.1038/ s41586-019-1156-9

Zhang P, McGrath B, Li S, Frank A, Zambito F, Reinert J, Gannon M, Ma K, McNaughton K, Cavener DR (2002) The PERK eukaryotic initiation factor 2 alpha kinase is required for the development of the skeletal system, postnatal growth, and the function and viability of the pancreas. Mol Cell Biol 22: 3864-3874. doi:10.1128/mcb.22.11.38643874.2002

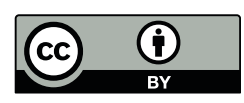

License: This article is available under a Creative Commons License (Attribution 4.0 International, as described at https://creativecommons.org/ licenses/by/4.0/). 